\title{
Graph Pattern Detection: Hardness for All Induced Patterns and Faster Non-induced Cycles
}

\author{
Mina Dalirrooyfard \\ MIT \\ Cambridge, MA, USA \\ minad@mit.edu
}

\author{
Thuy Duong Vuong \\ MIT \\ Cambridge, MA, USA \\ dvuong@mit.edu
}

\author{
Virginia Vassilevska Williams \\ MIT \\ Cambridge, MA, USA \\ virgi@mit.edu
}

\begin{abstract}
We consider the pattern detection problem in graphs: given a constant size pattern graph $H$ and a host graph $G$, determine whether $G$ contains a subgraph isomorphic to $H$. We present the following new improved upper and lower bounds:

We prove that if a pattern $H$ contains a $k$-clique subgraph, then detecting whether an $n$ node host graph contains a not necessarily induced copy of $H$ requires at least the time for detecting whether an $n$ node graph contains a $k$-clique. The previous result of this nature required that $H$ contains a $k$-clique which is disjoint from all other $k$-cliques of $H$.

We show that if the famous Hadwiger conjecture from graph theory is true, then detecting whether an $n$ node host graph contains a not necessarily induced copy of a pattern with chromatic number $t$ requires at least the time for detecting whether an $n$ node graph contains a $t$-clique. This implies that: (a) under Hadwiger's conjecture for every $k$-node pattern $H$, finding an induced copy of $H$ requires at least the time of $\sqrt{k}$-clique detection and size $\omega\left(n^{\sqrt{k} / 4}\right)$ for any constant depth circuit, and (b) unconditionally, detecting an induced copy of a random $G(k, p)$ pattern w.h.p. requires at least the time of $\Theta(k / \log k)$-clique detection, and hence also at least size $n^{\Omega(k / \log k)}$ for circuits of constant depth.

We show that for every $k$, there exists a $k$-node pattern that contains a $k-1$-clique and that can be detected as an induced subgraph in $n$ node graphs in the best known running time for $k-1$-Clique detection. Previously such a result was only known for infinitely many $k$.

Finally, we consider the case when the pattern is a directed cycle on $k$ nodes, and we would like to detect whether a directed $m$-edge graph $G$ contains a $k$-Cycle as a not necessarily induced subgraph. We resolve a 14 year old conjecture of [Yuster-Zwick SODA'04] on the complexity of $k$-Cycle detection by giving a tight analysis of their $k$-Cycle algorithm. Our analysis improves the best bounds for $k$-Cycle detection in directed graphs, for all $k>5$.
\end{abstract}

Permission to make digital or hard copies of all or part of this work for personal or classroom use is granted without fee provided that copies are not made or distributed for profit or commercial advantage and that copies bear this notice and the full citation on the first page. Copyrights for components of this work owned by others than ACM must be honored. Abstracting with credit is permitted. To copy otherwise, or republish, to post on servers or to redistribute to lists, requires prior specific permission and/or a fee. Request permissions from permissions@acm.org.

STOC '19, June 23-26, 2019, Phoenix, AZ, USA

(c) 2019 Association for Computing Machinery.

ACM ISBN 978-1-4503-6705-9/19/06 \$15.00

https://doi.org/10.1145/3313276.3316329

\section{CCS CONCEPTS}

- Theory of computation $\rightarrow$ Graph algorithms analysis; Problems, reductions and completeness.

\section{KEYWORDS}

Fine-grained complexity, Subgraph isomorphism, Cliques, Cycle detection

\section{ACM Reference Format:}

Mina Dalirrooyfard, Thuy Duong Vuong, and Virginia Vassilevska Williams. 2019. Graph Pattern Detection: Hardness for All Induced Patterns and Faster Non-induced Cycles. In Proceedings of the 51st Annual ACM SIGACT Symposium on the Theory of Computing (STOC '19), June 23-26, 2019, Phoenix, AZ, USA. ACM, New York, NY, USA, 12 pages. https://doi.org/10.1145/3313276. 3316329

\section{INTRODUCTION}

One of the most fundamental graph algorithmic problems is Subgraph Isomorphism: given two graphs $G=(V, E)$ and $H=\left(V_{H}, E_{H}\right)$, determine whether $G$ contains a subgraph isomorphic to $H$. While the general problem is NP-complete, many applications (e.g. from biology $[2,31]$ ) only need algorithms for the special case in which $H$ is a small graph pattern, of constant size $k$, while the host graph $G$ is large. This graph pattern detection problem is easily in polynomial time: if $G$ has $n$ vertices, the brute-force algorithm solves the problem in $O\left(n^{k}\right)$ time, for any $H$.

Two versions of the Subgraph Isomorphism problems are typically considered. The first is the induced version in which one seeks an injective mapping $f: V_{H} \mapsto V$ so that $(u, v) \in E_{H}$ if and only if $(f(u), f(v)) \in E$. The second is the not necessarily induced version where one seeks an injective mapping $f: V_{H} \mapsto V$ so that if $(u, v) \in E_{H}$ then $(f(u), f(v)) \in E$ (however, if $(u, v) \notin E_{H}$, $(f(u), f(v))$ may or may not be an edge). It is not hard to show (e.g. via color-coding) that when $k$ is a constant, any algorithm for the induced version can be used to solve the not necessarily induced one (for the same pattern) in asymptotically the same time, up to logarithmic factors.

This paper considers two settings of the graph pattern detection problem: (1) Finding induced patterns of constant size $k$ in dense $n$-node undirected graphs, where the runtime is measured as a function of $n$, and (2) Finding not-necessarily induced patterns in sparse $m$-edge directed graphs; here we focus on $k$-Cycle patterns, a well-studied and important case. 


\subsection{Hardness for all Induced and Some not Necessarily Induced Patterns.}

A standard generalization of a result of Nešetril and Poljak [29] shows that the induced subgraph isomorphism problem for any $k$ node pattern $H$ in an $n$-node host graph can be reduced in $O\left(k^{2} n^{2}\right)$ time to the $k$-Clique (or induced $k$-Independent Set (IS)) detection problem in $k n$-node graphs. Thus, for constant $k, k$-Clique and $k$-IS are the hardest patterns to detect.

Following Itai and Rodeh [21], Nešetril and Poljak [29] showed that a $k$-Clique (and hence any induced or not-necessarily induced $k$-node pattern) can be detected in an $n$ node graph $G$ asymptotically in time $C(n, k):=M\left(n^{\lfloor k / 3\rfloor}, n^{\lceil k / 3\rceil}, n^{\lceil(k-1) / 3\rceil}\right)$, where $M(a, b, c)$ is the fastest known runtime for multiplying an $a \times b$ by a $b \times c$ matrix. A simple bound for $M(a, b, c)$ is $M(a, b, c) \leq a b c / \min \{a, b, c\}^{3-\omega}$ where $\omega<2.373$ is the exponent of square matrix multiplication [26, 39], but faster algorithms are known (e.g. Le Gall and Urrutia [18]). In particular, $C(n, k) \leq O\left(n^{\omega k / 3}\right)$ when $k$ is divisible by 3 .

The $C(n, k)$ runtime for $k$-Clique detection has had no improvements in more than 40 years. Because of this, several papers have hypothesized that the runtime might be optimal for $k$-Cliques (and $k$-Independent Sets) (e.g. $[1,8,27])$.

Meanwhile, for some $k$-node patterns $H$ that are not Cliques or Independent Sets, specialized algorithms have been developed that are faster than the $C(n, k)$ runtime for $k$-Clique. For instance, if $H$ is a 3-node pattern that is not a triangle or an independent set, it can be detected in $G$ in linear time, much faster than the $C(n, 3)=O\left(n^{\omega}\right)$ time for 3-Clique/triangle. Following work of [10, 14, 23, 25, 30], Vassilevska W. et al. [41] showed that every 4-node pattern except for the 4-Clique and 4-Independent Set can be detected in $C(n, 3)=O\left(n^{\omega}\right)$ time, much faster than the $C(n, 4)$ runtime for 4-Clique. Blaser et al. [4] recently showed that for $k \leq 8$ there are faster than $C(n, k)$ time algorithms for all non-clique non-independent set $k$-node patterns; for $k \leq 6$, their runtime is $C(n, k-1)$. Independently, we were able to show the same result, using an approach generalizing ideas from [41] (see [13]).

A natural conjecture, consistent with the prior work so far is that for every $k$ and every $k$-node induced pattern $H$ that is not a clique or independent set, one can detect it in an $n$ node graph in time $C(n, k-1)$. Blaser et al. showed that for all $k$ of the form $3 \cdot 2^{\ell}$ for integer $\ell$, there is a $k$-node induced pattern that (1) is at least as hard to detect as $k-1$-Clique and (2) can be detected in $C(n, k-1)$ time. We show that such a pattern exists for all $k \geq 3$ (Theorem 3.2).

While there exist $k$-node induced patterns that can be detected faster than $k$-Clique, it seems unclear how hard $k$-node pattern detection actually is. For instance, it could be that for every $k$, there is some induced pattern on $k$-nodes that can be detected in say $n^{\log \log (k)}$ time. A Ramsey theoretic result tells us that every $k$-node $H$ either contains an $\Omega(\log k)$ size clique or an $\Omega(\log k)$ size independent set. Hence intuitively, detecting any $k$-node induced $H$ in an $n$ node graph should be at least as hard as detecting an $\Omega(\log k)$ size clique in an $n$-node graph. The widely believed Exponential Time Hypothesis (ETH) [20] is known to imply that $k$-Clique cannot be solved in $n^{o(k)}$ time [9]. Coupled with the Ramsey result, ETH should intuitively imply that no matter which $k$-node $H$ we pick, $H$-pattern detection cannot be solved in $n^{o(\log k)}$ time.
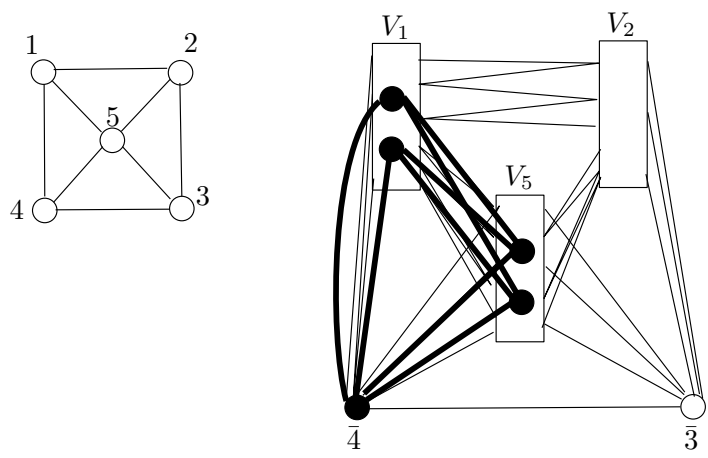

Figure 1: An example of how a simple reduction attempt fails to reduce 3-Clique to $H$. The edges between the $V_{i}$ are determined by the 3-Clique instance.

Unfortunately, however, it is still open whether every pattern that contains a $t$-clique is as hard to detect as a $t$-clique (see e.g. [4] ${ }^{1}$ ). In general, it is not clear what makes patterns hard to detect.

One of the few results related to this is by Floderus et al. [16] who showed that if a pattern $H$ contains a $t$-Clique that is disjoint from all other $t$-Cliques in $H$, then $H$ is at least as hard to detect as a $t$-Clique. This implied strong clique-based hardness results for induced $k$-path and $k$-cycle. However, the reduction of [16] fails for patterns whose $k$-Cliques intersect non-trivially.

The main difficulty in reducing $k$-Clique to the detection problem for other graph patterns $H$ can be seen in the following natural attempt used e.g. by [16]. Say $H$ has a $k$-clique $K$ and let $H^{\prime}$ be the graph induced by the vertices of $H$ not in $K$. Let $G=(V, E)$ be an instance of $k$-Clique. We'll start by creating $k$ copies of $V$, $V_{1}, \ldots, V_{k}$. For every edge $(u, v)$ of $G$, add an edge between the copies of $u$ and $v$ in different parts. Every $k$-clique $C$ of $G$ appears in the new graph $k$ ! times; we'll say that the main copy $\bar{C}$ of $C$ has the $i$ th vertex of $C$ (in lexicographic order say) appearing in $V_{i}$. Now, add a copy $\bar{H}^{\prime}$ of $H^{\prime}$, using fresh vertices, and for every edge $(h, i)$ of $H$ with $h \in H^{\prime}$ and $i \in K$, add edges from $h \in \bar{H}^{\prime}$ to all vertices in $V_{i}$. This forms the new graph $G^{\prime}$ and guarantees that if $G$ has a $k$-clique $C, G^{\prime}$ contains a copy of $H$ which is just $\bar{C}$ together with $\bar{H}^{\prime}$. The other direction of the reduction fails miserably however. If $G^{\prime}$ happens to have a copy of $H$, there is no guarantee that any of the $k$-cliques of $H$ would have a node from each $V_{i}$ and hence form a clique of $G$. As a simple counterexample (Figure 1) consider $H$ as a 4 -Cycle $(1,2,3,4)$ together with a node 5 that has edges to all nodes of the 4-Cycle. Starting from a graph $G$, WLOG we would pick $K$ to be $(1,2,5)$ and $H^{\prime}=3,4$ and form $G^{\prime}$ as described. Let $\bar{H}^{\prime}$ contain the nodes $\overline{3}, \overline{4}$ and let the parts of $G$ be $V_{1}, V_{2}, V_{5}$. Now the reduction graph $G^{\prime}$ might contain a copy of $H$ even if $G$ has no 3-cliques, as $\overline{4}$ could represent 5 , and 1, 3 and 2, 4 could be represented by two nodes each in $V_{1}$ and $V_{5}$ respectively; see Figure 1 . Hence the copy of $H$ wouldn't use $V_{2}$ at all and doesn't represent a triangle in $G$.

One could try to modify the reduction, say by representing the nodes of $H^{\prime}$ by copies of the vertices of $G$, as with $K$. However,

\footnotetext{
${ }^{1}$ Blaser et al. [4] show that for the particular types of algorithms that they use a pattern that contains a $k$-clique cannot be found faster than a $k$-clique, and they note that such a result is not known for arbitrary algorithms.
} 
the same issues arise, and they seem to persist in most natural reduction attempts.

With an intricate construction, we show how to overcome this difficulty. Our first main theorem is that patterns that contain $t$ cliques are indeed at least as hard as $t$-Clique, and in fact we prove it for the not necessarily induced case which automatically gives a lower bound for the induced case:

Theorem 1.1. Let $G=(V, E)$ be an n-node, $m$-edge graph and let $H$ be a $k$-node pattern such that $H$ has a t-clique as a subgraph. Then one can construct a new graph $G^{*}$ of at most $n k$ vertices in $O\left(k^{2} m+k n\right)$ time such that $G^{*}$ has a not necessarily induced subgraph isomorphic to $H$ if and only if $G$ has a $t$-clique.

Thus ETH indeed implies that no matter which $k$-node $H$ we pick, $H$-pattern detection cannot be solved in $n^{o(\log k)}$ time.

Our second theorem shows that some patterns are even harder, as in fact the hardness of a pattern grows with its chromatic number!

Our theorem relies on the widely believed Hadwiger conjecture [19] from graph theory which roughly states that every graph with chromatic number $t$ contains a $t$-clique as a minor. The Hadwiger conjecture is known to hold for $t \leq 6$ [33] and to almost hold for $t=7$ [22] (It is equivalent to the 4-Color Theorem for $t=5,6[32,33,40]$.). It also holds for almost all graphs [6]. Our lower bound theorem, which is also proved for the not necessarily induced case is:

Theorem 1.2. Let $G=(V, E)$ be an n-node graph and let $H$ be a $k$-node pattern with chromatic number $t$, for $t>1$. Then assuming that Hadwiger conjecture is true, one can construct $G^{*}$ on at most $n k$ vertices in $O\left(n^{2} k^{2}\right)$ time such that $G^{*}$ has a not necessarily induced subgraph isomorphic to $H$ if and only if $G$ has a $t$-clique.

This is the first connection between the Hadwiger conjecture and Subgraph Isomorphism, to our knowledge. Let us see some exciting consequences of this theorem. First, we get that if $t$ is the maximum of the chromatic numbers of $H$ and its complement, then an induced $H$ is at least as hard as $t$-Clique to detect. Now, it is a simple exercise that the maximum of the chromatic number of a $k$-node graph and its complement is at least $\sqrt{k}$. Thus, every induced $H$ on $k$-nodes is at least as hard as $\sqrt{k}$-Clique. There are no easy patterns.

COROLLARY 1.1. No matter what $k$-node $H$ we take, under ETH and the Hadwiger Conjecture, the induced subgraph isomorphism problem for $H$ in $n$-node graphs cannot be solved in $n^{o(\sqrt{k})}$ time.

This is the first result of such generality.

A second consequence comes from circuit complexity. Rossman [34] showed that for any constant $k$, any constant depth circuit requires size $\omega\left(n^{k / 4}\right)$ to detect a $k$-Clique. Because of the simplicity of our reduction (it can be implemented in constant depth), we also obtain a circuit lower bound for induced pattern detection for any $H$ node subgraph: No matter what $k$-node $H$ we take, under the Hadwiger Conjecture, any constant depth circuit for the induced subgraph isomorphism problem for $H$ in $n$-node graphs requires size $\omega\left(n^{\sqrt{k} / 4}\right)$.

A third consequence is that in fact almost all $k$-node patterns are very hard - at least as hard as $\Theta(k / \log k)$-Clique. Consider an ErdösRenyi graph $H$ from $G(k, p)$ for constant $p$. It is known [6] that the
Hadwiger conjecture holds for $H$ with high probability. Moreover, the chromatic number of such graphs (and their complements) is with high probability $\Theta(k / \log k)$ [5]; meanwhile the clique and independent set size is only $O(\log k)$. Thus our chromatic number theorem significantly strengthens our first theorem.

Corollary 1.2. For almost all $k$-node patterns $H$, under ETH, induced $H$ detection in $n$ node graphs cannot be done in $n^{o(k / \log k)}$ time.

We also immediately obtain, via Rossman's lower bound, that for almost all $k$-node patterns $H$, any constant depth circuit that can detect an induced $H$ requires size $n^{\Omega(k / \log k)}$.

\subsection{Detecting Not-Necessarily Induced Directed k-Cycles.}

Some of the most striking difference between the complexity of induced and not-necessarily induced subgraph detection is in the $k$-Path and $k$-Cycle problems. Since a $k$-Path and a $k$-Cycle both contain an independent set on $\lfloor k / 2\rfloor$ nodes, the induced version of their subgraph detection problems is at least as hard as detecting $\lfloor k / 2\rfloor$-cliques, and needs $C(n,\lfloor k / 2\rfloor)$ time unless there is a breakthrough in Clique detection. Thus also under ETH, induced $k$-Path and $k$-Cycle cannot be solved in $n^{o(k)}$ time. Monien [28], however, showed that for all constants $k$, a non-induced $k$-Path can be detected with constant probability in linear time. Thus, for constant $k$, the non-induced $k$-Path problem has an essentially optimal (randomized) algorithm. With the same ideas, a $k$-Cycle can be found in $\tilde{O}\left(n^{\omega}\right)$ time. Due to the tight relationship between triangle detection and Boolean matrix multiplication (e.g. [37]), this runtime is often conjectured to be optimal for dense graphs. For sparse graphs, however, there has been a lot of active research in improving the runtime of $k$-Cycle detection, and it is completely unclear what the best runtime should be.

Alon, Yuster and Zwick [3] gave several algorithms for both directed and undirected cycle detection. The bounds for directed graphs are as follows. For 3-Cycles (triangles) [3] gives an algorithm running in time $O\left(m^{2 \omega /(\omega+1)}\right) \leq O\left(m^{1.41}\right)$, which is still the fastest algorithm for the problem in sparse graphs. For general $k$, one can find a $k$-Cycle in time $O\left(\mathrm{~m}^{2-2 / k}\right)$ if $k$ is even and in time $O\left(m^{2-2 /(k+1)}\right)$ if $k$ is odd. These last algorithms do not use matrix multiplication.

Yuster and Zwick [43] set out to improve upon the general $k$ Cycle algorithms above using fast matrix multiplication. They presented an algorithm that combines most known techniques for cycle detection and works for arbitrary $k \geq 3$. However, they were not able to analyze the complexity of their algorithm in general. They showed that for $k=4$, the algorithm runs in $O\left(m^{(4 \omega-1) /(2 \omega+1)}\right) \leq$ $O\left(m^{1.48}\right)$ time, and that for $k=5$, it runs in time $O\left(m^{3 \omega /(\omega+2)}\right) \leq$ $O\left(m^{1.63}\right)$. Both bounds improve the runtimes from [3].

Already for $k=6$ the analysis of the algorithm seemed very difficult. Yuster and Zwick ran computer simulations to find the worst case runtime for $k=6$ and beyond and came up with conjectures for what the runtime should be for $k=6$ and for all odd $k$. They also stated that for even $k$ larger than 6 , the runtime expression is likely extremely complicated. Their conjectures have remained unproven for over 14 years. 
In this paper we present an analysis of the running time of the Yuster-Zwick algorithm, proving the two conjectures (for $k=6$ and odd $k$ ). We give an analysis of the runtime for even $k$ as well. Our bound is tight, assuming that the matrix multiplication exponent $\omega$ is 2. For larger values of $\omega$, the tight bound on the runtime is a step function of $\omega$ that remains to be analyzed. Our final result is as follows:

THEOREM 1.3. There is an algorithm for $k$-Cycle detection in $m$ edge directed graphs (the Yuster-Zwick algorithm) which runs in $\tilde{\Theta}\left(m^{c_{k}}\right)$ time, where

- $c_{k}=\omega(k+1) /(2 \omega+k-1)$ when $k$ is odd,

- $c_{4}=(4 \omega-1) /(2 \omega+1)$

-

$$
c_{6}= \begin{cases}\frac{10 \omega-3}{4 \omega+3}, & \text { if } 2 \leq \omega \leq \frac{13}{6} \\ \frac{22-4 \omega}{17-4 \omega}, & \text { if } \frac{13}{6} \leq \omega \leq \frac{9}{4} \\ \frac{11 \omega-2}{4 \omega+5}, & \text { if } \frac{9}{4} \leq \omega \leq \frac{16}{7} \\ \frac{10-\omega}{7-\omega}, & \text { if } \frac{16}{7} \leq \omega \leq \frac{5}{2}\end{cases}
$$

- $c_{k} \leq(k \omega-4 / k) /(2 \omega+k-2-4 / k)$ for all even $k \geq 4$. This is tight for $\omega=2$.

Related Work. Vassilevska [38] showed that $K_{k}-e$ (a $k$-clique missing an edge) can be found in $O\left(n^{k-1}\right)$ time without using fast matrix multiplication, whereas the fastest algorithms for $k$-Clique without fast matrix multiplication run in $O\left(n^{k} / \log ^{k-1} n\right)$ time [36]; this was recently improved by Blaeser et al. [4] who showed that every $k$ node pattern except the $k$-Clique and $k$-Independent Set can be detected in time $O\left(n^{k-1}\right)$. Before this, Floderus et al. [15] showed that 5 node patterns ${ }^{2}$ can be found in $O\left(n^{4}\right)$ time, again without using fast matrix multiplication.

Some other related work includes improved algorithms for subgraph detection when $G$ has special structure (e.g. [24] and [17]). Other work counts the number of occurrences of a pattern in a host graph (e.g. [11, 25, 35]). Finally, there is some work on establishing conditional lower bounds. Floderus et al. [16] produced reductions from $k$-Clique (or $k$-Independent Set) to the detection problem of $\ell$-patterns for $\ell>k$ (but still linear in $k$ ). They show for instance that finding an induced $k$-path is at least as hard as finding an induced $k / 2$-independent set. Lincoln et al. [27] give conditional lower bounds for not-necessarily induced directed $k$-cycle detection. For instance, they show that if $k$-Clique requires essentially $C(n, k)$ time, then finding a directed $k$-Cycle in an $m$ edge graph requires $m^{2 \omega k /(3(k+1))-o(1)}$ time. This lower bound is lower than the upper bounds in this paper, but it does show that superlinear time is likely needed.

Detecting $k$-Cycles in undirected graphs is an easier problem, when $k$ is an even constant. Yuster and Zwick [42] showed that a $k$-Cycle in an undirected graph can be detected (and found) in $O\left(n^{2}\right)$ time for all even constants $k$. Dahlgaard et al. [12] extended this result showing that $k$-Cycles for even $k$ in $m$-edge graphs can be found in time $\tilde{O}\left(\mathrm{~m}^{2 k /(k+1)}\right)$. Their result implies that of [42], as by a result of Bondy and Simonovits [7], any $n$ node graph with $\geq 100 k n^{1+1 / k}$ edges must contain a $2 k$-Cycle. When $k$ is an odd

\footnotetext{
${ }^{2}$ All patterns except for $K_{5}, K_{4}+e,(3,2)$-fan, gem, house, butterfly, bull, $C_{5}, K_{1,4}, K_{2,3}$ and their complements; for these subgraphs the fastest runtime remained $C(n, 5) \leq$ $O\left(n^{4.09}\right)$.
}

constant, the $k$-Cycle problems in undirected and directed graphs are equivalent (see e.g. [38]).

\section{LOWER BOUNDS}

In this section we consider the problem of detecting and finding a (not necessarily induced) copy of a given small pattern graph $H$ in a host graph $G$. This is the variant of subgraph isomorphism in which the pattern $H$ is fixed, on a constant $k$ number of vertices, and $G=(V, E)$ with $|V|=n$ is given as an input. We focus on the hardness of this problem: we show that any fixed pattern that has a $t$-clique as a subgraph, is not easier to detect as a subgraph than a $t$-clique, formally stated as Theorem 1.1. First, we start by an easier case of the theorem where the pattern is $t$-chromatic to depict the main idea of our proof and then we proceed with the proof of the theorem for all patterns. Recall that a proper vertex coloring of a graph is an assignment of colors to each of its vertices such that no edge connects two identically colored vertices. If the set of colors is of size $c$, we say that the graph is $c$-colorable. The chromatic number of a graph is the smallest number $c$ for which the graph is $c$-colorable, and we call such graph c-chromatic. In the second part of this section, we prove a stronger lower bound, showing that under the Hadwiger conjecture, any $t$-chromatic pattern is not easier to detect as a subgraph than a $t$-clique.

\subsection{Simple Case: $t$-Chromatic Patterns}

We show Theorem 1.1 when $H$ is $t$-chromatic in addition to having a $t$-clique as a subgraph. Construct $G^{*}$ as follows: For each $v \in H$, let $G_{v}$ be a copy of the vertices of $G$ as an independent set. For any two vertices $v$ and $u$ in $H$ where $v u$ is an edge, add the following edges between $G_{v}$ and $G_{u}$ : for each $w_{1}$ and $w_{2}$ in $G$, add an edge between the copy of $w_{1}$ in $G_{v}$ and the copy of $w_{2}$ in $G_{u}$ if and only if $w_{1} w_{2}$ is an edge in $G$. So $G^{*}$ has $n k$ vertices and since for each pair of vertices $u, v \in H$ we have at most $m$ edges between $G_{u}$ and $G_{v}$, the construction time is at most $O\left(k^{2} m+k n\right)$.

Now we show that $G$ has a $t$-clique as a subgraph if and only if $G^{*}$ has $H$ as a subgraph. First suppose that $G$ has a $t$-clique, say $T=v_{1}, \ldots, v_{t}$. Consider a $t$-coloring of the vertices of $H$, with colors $1, \ldots, t$. For each $w \in H$, pick $v_{i}$ from $G_{w}$ if $w$ is of color $i$. Call the induced subgraph on these vertices $H^{*}$. We show that $H^{*}$ is isomorphic to $H$ : map each $w \in H$ to the vertex picked from $G_{w}$ in $G^{*}$. If $w$ and $w^{\prime}$ are adjacent in $H$, then their colors are different, so the vertices that are picked from $G_{w}$ and $G_{w^{\prime}}$ are different vertices of $G$, and they are part of the clique $T$, so they are adjacent. If $w$ and $w^{\prime}$ are not adjacent, we don't have any edges between $G_{w}$ and $G_{w^{\prime}}$, so the vertices picked from them are not adjacent.

For the other direction, we show that if $G^{*}$ has $H$ as a subgraph then $G$ has a $t$-clique. Since $H$ has a $t$-clique as a subgraph, $G^{*}$ also has a $t$-clique as a subgraph. Suppose the vertices of this clique are $W=\left\{w_{1}, \ldots, w_{t}\right\}$ where $w_{i}$ is a copy of $v_{i} \in G$. Each pair of vertices of the clique are in different copies of $G$, as these copies are independent sets. Moreover, for each $i, j \in\{1, \ldots, t\}$, since $w_{i}$ and $w_{j}$ are adjacent, they correspond to different vertices in $G$, so $v_{i} \neq v_{j}$. Since we connect two vertices in $G^{*}$ if their corresponding vertices in $G$ are connected, this means that $v_{i}$ and $v_{j}$ are connected in $G$. So $v_{1}, \ldots, v_{t}$ form a $t$-clique in $G$. 

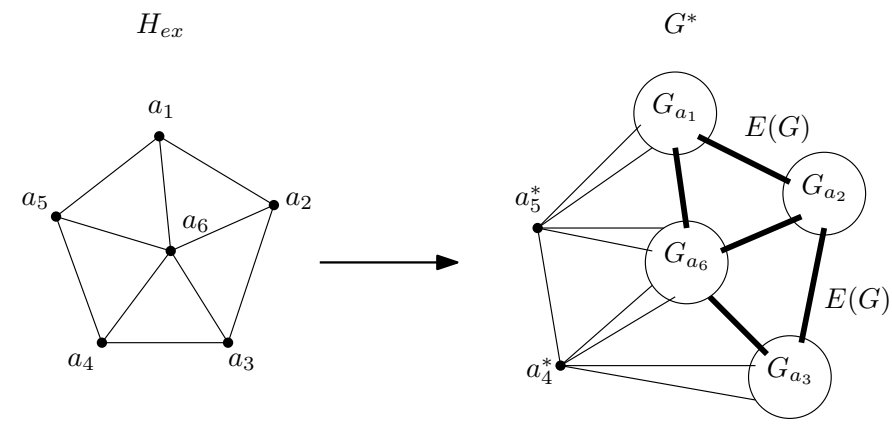

Figure 2: Graph $H_{e x}$ on the left. The largest clique of this graph is a triangle. $H_{e x}$ is 4-chromatic, so $p\left(H_{e x}\right)>1$. We have $p\left(H_{e x}\right)=2$, as a minimum 3-clique covering for it is $\left\{\left\{a_{1}, a_{2}, a_{3}, a_{6}\right\},\left\{a_{3}, a_{4}, a_{5}, a_{1}, a_{6}\right\}\right\}$. The graph $G^{*}$ is on the right, thick edges represent the way the edges are specified according to $E(G)$ between two copies of $G$.

\subsection{General Case}

Define a $t$-clique covering of a pattern $H$ to be a collection $C$ of sets of vertices of $H$, such that the induced subgraph on each set is $t$-colorable, and for any $t$-clique $T$ of $H$, there is a set in $C$ that contains all the vertices of $T$. See figure 2 for an example.

For each $H$ we have at least one $t$-clique covering by considering the vertices of each $t$-clique of $H$ as one set. However we are interested in the smallest collection $C$. So for a fixed $t$, we define $p(H)$ to be the smallest integer $r \geq 1$, such that there is a $t$-clique covering of $H$ of size $r$, and we call such covering a minimum $t$ clique covering. For example, if $H$ is $t$-colorable, $p(H)=1$ as the whole vertex set is the only set that the $t$-clique covering has. If $H$ is not $t$-colorable but has a $t$-clique, then $p(H)>1$.

Proof of Theorem 1.1. Let $C=\left\{C_{1}, \ldots, C_{r}\right\}$ be a minimum $t$-clique covering of $H$, where $r=p(H)$. The vertex set of $G^{*}$ is the following: For each vertex $v \in C_{1}$, let $G_{v}$ be a copy of the vertices of $G$ as an independent set. For each vertex $v \in V(H) \backslash C_{1}$, let $v^{*}$ be a copy of $v$ in $G^{*}$. The edge set of $G^{*}$ is as follows: For each two vertices $v, u \in C_{1}$ that $u v$ is an edge in $H$, add the following edges between $G_{v}$ and $G_{u}$ : for each $w_{1}$ and $w_{2}$ in $G$, add an edge between the copy of $w_{1}$ in $G_{v}$ and the copy of $w_{2}$ in $G_{u}$ if and only if $w_{1} w_{2}$ is an edge in $G$. For each two vertices $u \in C_{1}$ and $v \in V(H) \backslash C_{1}$ that $u v$ is an edge in $H$, connect $v^{*}$ to all the vertices in $G_{u}$. For each two vertices $u, v \in V(H) \backslash C_{1}$ that $u v$ is an edge in $H$, connect $u^{*}$ and $v^{*}$. The way $G^{*}$ is constructed is shown in Figure 2 for the particular pattern $H_{e x}$ with maximum clique 3 .

Now we show that $G$ has a $t$-clique as a subgraph if and only if $G^{*}$ has $H$ as a subgraph. First suppose that $G$ has a $t$-clique, say $v_{1}, \ldots, v_{t}$. Consider a $t$-coloring of vertices of $C_{1}$, with colors $1, \ldots, t$. Let $H^{*}$ be the subgraph on the following vertices in $G^{*}$ : for each $w \in C_{1}$, pick $v_{i}$ from $G_{w}$ if $w$ is of color $i$. For each $w \in V(H) \backslash C_{1}$, pick $w^{*}$. We show that $H$ is isomorphic to $H^{*}$ : for each $w \in C_{1}$, map $w$ to the vertex picked from $G_{w}$, and for each $w \in V(H) \backslash C_{1}$, map $w$ to $w^{*}$. If $w, u \in C_{1}$ such that $w u \in E(H)$, then their colors are different in the $t$-coloring of $C_{1}$, and so the vertices that are picked from $G_{w}$ and $G_{u}$ are different vertices of $G$ and part of the $t$-clique of $G$, so they are attached. If $w u$ is not an edge, then there is no edge between $G_{w}$ and $G_{u}$. If $w \in C_{1}$ and $u \in V(H) \backslash C_{1}$ and $w u$ is an edge in $H$, then $u^{*}$ is attached to all vertices in $G_{w}$ including the vertex that is picked from $G_{w}$ for $H^{*}$. If $w u$ is not an edge, then there is no edge between $u^{*}$ and $G_{w}$. If $w, u \in V(H) \backslash C_{1}$, then $u^{*}, w^{*}$ are both picked in $H^{*}$ and they are adjacent in $G^{*}$ if and only if $w$ and $u$ are adjacent in $H$.

For the other direction, we show that if $G^{*}$ has a subgraph $H^{*}$ isomorphic to $H$, then $G$ has a $t$-clique. Let $S_{1}=\cup_{v \in C_{1}} G_{v}$. First suppose that $H^{*}$ has a $t$-clique $T$ using vertices in $S_{1}$. Since for each $v \in C_{1}, G_{v}$ is an independent set, no two vertices of $T$ are in the same $G_{v}$. So there are $t$ vertices of $H, v_{1}, \ldots, v_{t}$ such that $T$ has a vertex in each $G_{v_{i}}$. Let this vertex be a copy of $w_{i} \in G$. Since for each $i, j \in\{1, \ldots, t\}, i \neq j$, the copies of $w_{i}$ and $w_{j}$ are adjacent in $G^{*}$, we have that $w_{i} \neq w_{j}$ and they are adjacent in $G$. So $\left\{w_{1}, \ldots, w_{t}\right\}$ form a $t$-clique in $G$.

So assume that the induced subgraph on $V\left(H^{*}\right) \cap S_{1}$ in $G^{*}$ has no clique. As $S_{1}$ has all the vertices in $G^{*}$ that correspond to the vertices in $C_{1}$, we define similar sets for other $C_{i}$ s. For $i \in\{2, \ldots, p(H)\}$, let $S_{i}^{\prime}=\cup_{v \in C_{i} \cap C_{1}} G_{v}, S_{i}^{\prime \prime}=\cup_{v \in C_{i} \backslash C_{1}} v^{*}$ and $S_{i}=S_{i}^{\prime} \cup S_{i}^{\prime \prime}$. First note that the induced subgraph on $S_{i}$ is $t$-colorable: Consider the $t$-coloring of $C_{i}$. For each $v \in C_{i} \backslash C_{1}$, color $v^{*}$ the same as $v$. For each $v \in C_{i} \cap C_{1}$, color all vertices in $G_{v}$ the same as $v$.

Now we show that any $t$-clique in $H^{*}$ is in one of the sets $S_{2}, \ldots, S_{p(H)}$. This means that the collection $\left\{S_{2} \cap V\left(H^{*}\right), \ldots, S_{p(H)} \cap\right.$ $\left.V\left(H^{*}\right)\right\}$ is a $t$-clique covering for $H$ with size $p(H)-1$, which is a contradiction. Consider a $t$-clique $T=v_{1}, \ldots, v_{t}$ in $H^{*}$. Each $v_{i}$ is in one of the copies of $G$ or is a copy of a vertex in $H$. So for each $v_{i}$, there is some vertex $w_{i} \in H$, such that $v_{i} \in G_{w_{i}}$ and $w_{i} \in C_{1}$ if $v_{i} \in S_{1}$, or $v_{i}=w_{i}^{*}$ and $w_{i} \notin C_{1}$ if $v_{i} \notin S_{1}$. Since for each $i, j, v_{i}$ and $v_{j}$ are adjacent in $G^{*}$, this means that $w_{i}$ and $w_{j}$ are different vertices in $H$ and they are adjacent. So $W=\left\{w_{1}, \ldots, w_{t}\right\}$ forms a clique in $H$. Since $T \notin S_{1}$, wlog we can assume that $v_{1} \notin S_{1}$. So $w_{1} \notin C_{1}$. So the $t$-clique $W$ is not in $C_{1}$, and so it is in $C_{i}$, for some $2 \leq i \leq p(H)$. So, $T \in S_{i}$.

\subsection{A Stronger Lower Bound}

One of the oldest conjectures in graph theory is the Hadwiger conjecture which intoduces a certain structure for $t$-chromatic graphs. Assuming that this conjecture is true, we show that any fixed pattern with chromatic number $t$ is not easier to detect as an induced subgraph than a $t$-clique. This strengthens the previous lower bound because the size of the maximum clique of a pattern is at most its chromatic number, and moreover there are graphs with maximum clique of size 2 but large chromatic number.

Conjecture 1 (Hadwiger's Conjecture). Let H be a graph with chromatic numbert. Then one can find t disjoint connected subgraphs of $H$ such that there is an edge between every pair of subgraphs.

Contracting the edges within each of these subgraphs so that each subgraph collapses to a single vertex produces a $t$-clique as a minor of $H$. This is the property we are going to use to show that $H$ is at least as hard to detect as a $t$-clique. Our main theorem is stated as Theorem 1.2.

To prove Theorem 1.2, we use a similar approach as Theorem 1.1. The approach of Theorem 1.1 is covering the maximum cliques of the pattern by a collection of subgraphs. However, since in Theorem 1.2 the pattern doesn't necessarily have a $t$-clique, we cover another 
particular subgraph of the pattern, and hence we introduce a similar notion as $t$-clique covering for this subgraph.

Let $F$ be a graph with a vertex (not necessarily proper) coloring $C: V(F) \rightarrow\{1, \ldots, t\}$. We say that $F$ has a $K_{t}$ minor with respect to the coloring $C$ if the vertices of each color induce a connected subgraph and for every color there is an edge from one of the vertices of that color to one of the vertices of every other color. For example, in Figure 2, consider the following coloring for $H_{e x}$ : $C_{e x}:\left\{a_{1}, \ldots, a_{6}\right\} \rightarrow\{1, \ldots, 4\}$, where $C_{e x}\left(a_{1}\right)=C_{e x}\left(a_{2}\right)=1$, $C_{e x}\left(a_{3}\right)=C_{e x}\left(a_{4}\right)=2, C_{e x}\left(a_{5}\right)=3$ and $C_{e x}\left(a_{6}\right)=4$. Clearly $H_{e x}$ has a $K_{4}$ minor with respect to the coloring $C_{e x}$.

Let $F$ and $H$ be two fixed graphs, where $F$ is $t$-chromatic. We say that $H$ is $\left(K_{t}, F\right)$ minor colorable if there is a (not necessarily proper) coloring $C: V(H) \rightarrow\{1, \ldots, t\}$ such that any induced copy of $F$ in $H$ has a $K_{t}$ minor with respect to $C$. For example, in Figure 3, the graph $H_{e x}^{\prime}$ has graph $H_{e x}$ (Figure 2) as a 4-chromatic subgraph, and it is $\left(K_{4}, H_{e x}\right)$ minor colorable: There are exactly two copies of $H_{e x}$ in $H_{e x}^{\prime}$, one with vertex set $\left\{a_{1}, \ldots, a_{6}\right\}$ and one with vertex set $\left\{a_{1}, a_{4}, a_{5}, a_{6}, a_{7}, a_{8}\right\}$, and both have a $K_{4}$ minor with respect to the coloring given.

Let $H$ be a pattern and let $F$ be a $t$-chromatic subgraph of $H$. As a generalization to a $t$-clique covering of $H$, we define an $F$-covering of $H$ to be a collection $C$ of sets of vertices of $H$, such that the induced subgraph of each set is $\left(K_{t}, F\right)$ minor colorable, and each copy of $F$ is completely inside one of the sets in $C$.

For any graph $H$, we have at least one $F$-covering by considering the vertices of each copy of $F$ as one set where the $\left(K_{t}, F\right)$ minor colorablitiy of each set comes from Conjecture 1 . Similar to $t$-clique coverings we are interested in the smallest collection $C$ among all $F$-coverings. So for a fixed number $t$ and a $t$-chromatic subgraph $F$ of $H$, we define $p_{F}(H)$ to be the smallest integer $r \geq 1$, such that there is an $F$-covering of $H$ of size $r$. We call an $F$-covering of size $p_{F}(H)$ a minimum $F$-covering. Note that $p_{K_{t}}(H)=p(H)$. For example, in Figure 3, $p_{H_{e x}}\left(H_{e x}^{\prime}\right)=1$, according to the coloring given in the figure.

Now we are ready to prove Theorem 1.2.

Proof of Theorem 1.2. We are going to mimic the proof of Theorem 1.1, and so we are going to carefully choose a subgraph $F$ and consider the minimum $F$-covering of it.

Let $z$ be the largest integer such that every $(z-1)$-node subgraph of $H$ is $t-1$ colorable. Let $F$ be a $t$-chromatic subgraph of $H$ on $z$ nodes with maximum number of edges. Note that $F$ is an induced subgraph of $H$. In Figure 3, $H=H_{e x}^{\prime}$ is 4-chromatic and one can check that any subgraph on 6 vertices or less is 3 colorable. In this graph $z=7$ and $F=H_{\text {ex }}$.

Now suppose that $C=\left\{C_{1}, \ldots, C_{r}\right\}$ is a minimum $F$-covering of $H$, where $r=p_{F}(H)$. Let $f: C_{1} \rightarrow\{1, \ldots, t\}$ be a $\left(K_{t}, F\right)$ minor coloring of $C_{1}$. Define the vertex set of $G^{*}$ as follows: For each vertex $v \in C_{1}$, let $G_{v}$ be a copy of $G$ as an independent set. For each vertex $v \in V(H) \backslash C_{1}$, let $v^{*}$ be a copy of $v$ in $G^{*}$. The edge set of $G^{*}$ is as follows: For each pair of vertices $u, v \in C_{1}$, if $u v$ is not an edge in $H$ we don't add any edges between $G_{u}$ and $G_{v}$. If $u v$ is an edge and $f(u)=f(v)$, then add the following edges between $G_{u}$ and $G_{v}$ : For each $w \in G$, add an edge between the copy of $w$ in $G_{u}$ and the copy of $w$ in $G_{v}$ (So we have a complete matching between $G_{u}$ and $G_{v}$ ). If $u v$ is an edge and $f(u) \neq f(v)$, then add the following edges between $G_{u}$ and $G_{v}$ : for each $w_{1}$ and $w_{2}$ in $G$, add an edge between the copy of $w_{1}$ in $G_{u}$ and the copy of $w_{2}$ in $G_{v}$ if and only if $w_{1} w_{2}$ is an edge in $G$. For each pair of vertices $u \in C_{1}$ and $v \in V(H) \backslash C_{1}$ such that $u v$ is an edge in $H$, add an edge between $v^{*}$ and all vertices in $G_{u}$. For each pair of vertices $u, v \in V(H) \backslash C_{1}$ such that $u v$ is an edge in $H$, add an edge between $u^{*}$ and $v^{*}$ in $G^{*}$. In Figure $3, H_{e x}^{\prime}$ has a $H_{e x}$-covering of size 1 which is the whole graph.

Now we show that $G$ has a $t$-clique as a subgraph if and only if $G^{*}$ has $H$ as a subgraph. First, suppose that $G$ has a $t$-clique, say $T=v_{1}, \ldots, v_{t}$. Let $H^{*}$ be the induced subgrpah on the following vertices in $G^{*}$ : for each $w \in C_{1}$, pick $v_{i}$ from $G_{w}$ if $f(w)=i$. For each $w \in V(H) \backslash C_{1}$, pick $w^{*}$. We show that $H$ is isomorphic to $H^{*}$ : for each $w \in C_{1}$, map $w$ to the vertex picked from $C_{w}$, and for each $w \in V(H) \backslash C_{1}$, map $w$ to $w^{*}$. If $u, w \in C_{1}$ and they are not adjacent, then there is no edge between $G_{u}$ and $G_{w}$. If $u w$ is an edge in $H$, then if $f(u)=f(w)=i$, we picked $v_{i}$ from both $G_{u}$ and $G_{w}$ and hence there are adjacent (note that in this case the edges between $G_{u}$ and $G_{w}$ form a complete matching). If $f(u) \neq f(w)$, then the vertices that we picked from $G_{u}$ and $G_{w}$ are copies of different vertices of the clique $T$, and so they are adjacent in $G^{*}$. If $u \in C_{1}$ and $w \in V(H) \backslash C_{1}$ and $u w$ is an edge in $H$, then $w^{*}$ is attached to all vertices in $G_{u}$, so it is adjacent to the vertex chosen from $G_{u}$ for $H^{*}$. If $u w$ is not an edge, then there is no edge between $w^{*}$ and $G_{u}$. If $u, w \in V(H) \backslash C_{1}$, then $u^{*}$ and $w^{*}$ are connected in $H^{*}$ if and only if $u w$ are connected in $H$.

For the other direction, we show that if $G^{*}$ has a (not necessarily induced) subgraph $H^{*}$ isomorphic to $H$, then $G$ has a $t$-clique. Let $S_{1}=\cup_{v \in C_{1}} G_{v}$. First suppose that $H^{*}$ has a copy of $F$ in $S_{1}$. Let the vertices of this copy be $w_{1}, \ldots, w_{z}$. For each $w_{i}$ there is a vertex $v_{i} \in H$ such that $w_{i} \in G_{v_{i}}$. Now if for some $i \neq j, v_{i}=v_{j}$, then the induced subgraph on $\left\{v_{1}, \ldots, v_{z}\right\}$ has less than $z$ vertices, so it is $t-1$ colorable (using proper coloring). Now if we color $w_{i}$ the same color as $v_{i}$, we get a proper coloring of this copy of $F$ with $t-1$ colors, a contradiction to the chromatic number of $F$. So for each $i \neq j, v_{i} \neq v_{j}$. Now we show that the induced subgraph on $\left\{v_{1}, \ldots, v_{z}\right\}$ in $H$ is isomorphic to $F$. Call this subgraph $F^{\prime}$. We just showed that $\left|V\left(F^{\prime}\right)\right|=z$. Since there is no edge between $G_{v_{i}}$ and $G_{v_{j}}$ if $v_{i}$ and $v_{j}$ are not connected, we have that $F$ is a subgraph of $F^{\prime}$, and so $F^{\prime}$ is not $t-1$ colorable, and since it is a subgraph of $H$, it is $t$-chromatic. If $F$ and $F^{\prime}$ are not isomorphic, then $F^{\prime}$ has more edges than $F$, which is a contradiction. So $F$ and $F^{\prime}$ are isomorphic, and in particular $w_{i}$ and $w_{j}$ are adjacent if and only if $v_{i}$ and $v_{j}$ are adjacent. Suppose that $w_{i} \in G_{v_{i}}$ is the copy of $w_{i}^{\prime}$ in $G$. We show that $\left\{w_{1}^{\prime}, \ldots, w_{z}^{\prime}\right\}$ induces a $t$-clique in $G$. Consider the coloring $f$ on $C_{1}$. First note that if $v_{i}$ and $v_{j}$ are adjacent vertices such that $f\left(v_{i}\right)=f\left(v_{j}\right)$, then since $w_{i}$ and $w_{j}$ are adjacent, we have $w_{i}^{\prime}=w_{j}^{\prime}$. Since $F_{1}$ has a $K_{t}$ minor with respect to the coloring $f$, the subgraph that each color induces is connected, and so for each $v_{i}$ and $v_{j}$ with $f\left(v_{i}\right)=f\left(v_{j}\right)=a$ we have $w_{i}^{\prime}=w_{j}^{\prime}$. This means that all $w_{i}$ 's with $f\left(v_{i}\right)=a$ are copies of the same vertex, say $u_{a}$. Now take a pair of colors, $a, b \in\{1, \ldots, t\}$. There are vertices $v_{i}$ and $v_{j}$ such that $f\left(v_{i}\right)=a, f\left(v_{j}\right)=b$ and $v_{i} v_{j}$ is an edge in $H$. So $w_{i} w_{j}$ is an edge in $G^{*}$, and since $a \neq b, w_{i}^{\prime} \neq w_{j}^{\prime}$, and $w_{i}^{\prime} w_{j}^{\prime}$ is an edge in $G$. Since $w_{i}^{\prime}=u_{a}$ and $w_{j}^{\prime}=u_{b}$, we have that $u_{a}$ and $u_{b}$ are different vertices and they are adjacent in $G$. So $\left\{w_{1}^{\prime}, \ldots, w_{z}^{\prime}\right\}=\left\{u_{1}, \ldots, u_{t}\right\}$ induces a $t$-clique in $G^{*}$. 

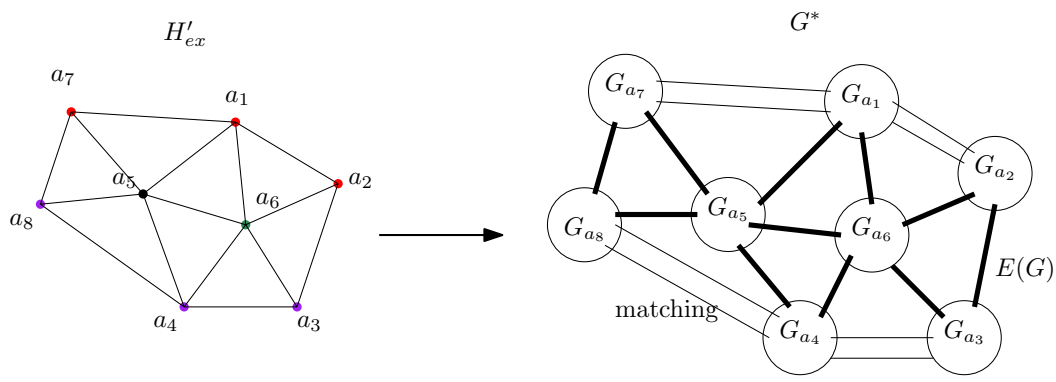

Figure 3: The 4-chromatic graph $H_{e x}^{\prime}$ on the left side has the coloring $C_{e x}^{\prime}$ which makes it $\left(K_{4}, H_{e x}\right)$ minor colorable: $C_{e x}^{\prime}\left(a_{1}\right)=$ $C_{e x}^{\prime}\left(a_{2}\right)=C_{e x}^{\prime}\left(a_{7}\right)=1, C_{e x}^{\prime}\left(a_{3}\right)=C_{e x}^{\prime}\left(a_{4}\right)=C_{e x}^{\prime}\left(a_{8}\right)=2, C_{e x}^{\prime}\left(a_{5}\right)=3, C_{e x}^{\prime}\left(a_{6}\right)=4$. On the right side we show how $G^{*}$ is constructed as it is described in the proof of Theorem 1.2. The double edges indicate a matching where nodes that are copy of the same vertex in $G$ are connected. The thick edges represent the way we add edges according to $E(G)$.

Now suppose that there is no copy of $F$ in the induced subgraph on $V\left(H^{*}\right) \cap S_{1}$ in $G^{*}$. For $i \in\left\{2, \ldots, p_{F}(H)\right\}$, let $S_{i}^{\prime}=\cup_{v \in C_{i} \cap C_{1}} G_{v}$,

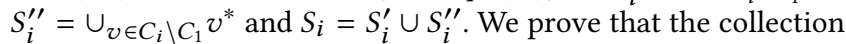
$\left\{S_{2} \cap V\left(H^{*}\right), \ldots, S_{p_{F}(H)} \cap V\left(H^{*}\right)\right\}$ is an $F$-covering for $H^{*}$, which means that $p_{F}(H)=p_{F}\left(H^{*}\right)<r$, a contradiction.

First we show that any copy of $F$ in $H^{*}$ is in one of $S_{i}$ s. Let $F^{*}$ with vertex set $\left\{w_{1}, \ldots, w_{z}\right\}$ be a copy of $F$ in $H^{*}$. For each $w_{i}$, there is a $v_{i} \in H$ where $w_{i} \in G_{v_{i}}$ and $v_{i} \in C_{1}$ if $w_{i} \in S_{1}$, or $w_{i}=v_{i}^{*}$ and $v_{i} \notin C_{1}$ if $w_{i} \notin S_{1}$. If $v_{i}=v_{j}$ for some $i \neq j$, then $F^{*}$ is $t-1$ colorable (with proper coloring): the induced graph on $\left\{v_{1}, \ldots, v_{z}\right\}$ has at most $z-1$ vertices and so it is $t-1$ colorable. Color $w_{i}$ the same as $v_{i}$. From the way we construct $G^{*}$ we know that if $v_{i}$ and $v_{j}$ are not connected, $w_{i}$ and $w_{j}$ are also not connected, and so this coloring of $F^{*}$ is proper. Since $F^{*}$ is $t$-chromatic, this is a contradiction. So if we call the induced graph on $\left\{v_{1}, \ldots, v_{z}\right\} \subseteq V(H)$ by $F^{H}$, then $\left|V\left(F^{H}\right)\right|=z$. We know that if $w_{i}$ and $w_{j}$ are connected, then $v_{i}$ and $v_{j}$ are connected. So $F$ is a subgraph of $F^{H}$, and so $F^{H}$ is $t$-chromatic. If $F^{H}$ and $F$ are not isomorphic, it means that $F^{H}$ has more edges than $F$, which is a contradiction. So $F^{H}$ and $F$ are isomorphic. Now Since $F^{*}$ is not in $S_{1}$, wlog we can assume that $w_{1} \notin S_{1}$, and so $w_{1}=v_{1}^{*}$ and $v_{1}$ is not in $C_{1}$. So $F^{H} \notin C_{1}$ and there is some $i \geq 2$ such that $F^{H} \in C_{i}$. So $F^{*}$ is in $S_{i}$.

Now we show that for each $i \geq 2, S_{i} \cap V\left(H^{*}\right)$ is $\left(K_{t}, F\right)$-minor colorable. Since $C_{i}$ is $\left(K_{t}, F\right)$-minor colorable, there is a coloring $f_{i}: C_{i} \rightarrow\{1, \ldots, t\}$ such that each induced copy of $F$ in $C_{i}$ has a $K_{t}$ minor with respect to $f_{i}$. Let $f_{i}^{*}: S_{i} \cap V\left(H^{*}\right) \rightarrow\{1, \ldots, t\}$ be the following coloring: For each $v \in C_{i} \cap C_{1}$, let $f_{i}^{*}(u)=f_{i}(v)$ for all vertices $u \in S_{i} \cap V\left(H^{*}\right) \cap G_{v}$. For each $v \in C_{i} \backslash C_{1}$ where $v^{*} \in V\left(H^{*}\right)$, let $f_{i}^{*}\left(v^{*}\right)=f_{i}(v)$. Now if $F^{*}=\left\{w_{1}, \ldots, w_{z}\right\}$ is a copy of $F$ in $S_{i} \cap V\left(H^{*}\right)$, we know that the set $F^{H}=\left\{v_{1}, \ldots, v_{z}\right\}$ is a copy of $F$ in $C_{i}$, where $w_{i} \in G_{v_{i}}$ if $w_{i} \in S_{1}$ and $w_{i}=v_{i}^{*}$ if $w_{i} \notin S_{1}$. Note that $f\left(v_{i}\right)=f_{i}^{*}\left(w_{i}\right)$ and $v_{i}$ and $v_{j}$ are adjacent if and only if $w_{i}$ and $w_{j}$ are adjacent. So since the subgraph induced on vertices of any color in $F^{H}$ is connected, the subgraph induced on any color in $F^{*}$ is also connected. Moreover, since in $f_{i}$ for any pair of colors there is an edge between one of the vertices of that color to one of the vertices of the other color, this property holds for $f_{i}^{*}$. So $S_{i} \cap V\left(H^{*}\right)$ is $\left(K_{t}, F\right)$-minor colorable, and so we have an $F$-covering for $H$ of size less than $p_{F}(H)$.

\section{INDUCED PATTERN DETECTION: ALGORITHMS}

In this section we focus on the algorithmic part of the induced pattern detection problem, starting with some background on the problem. First, it is a simple and folklore exercise to show that if there is a $T(n)$ time algorithm that can detect whether $G$ contains a copy of $H$, then one can also find such a copy in $O(T(n))$ time (see [13]). As finding and detection are equivalent, we will focus on the detection version of the problem. Recall from the introduction, $C(n, k):=M\left(n^{\lfloor k / 3\rfloor}, n^{\lceil k / 3\rceil}, n^{\lceil(k-1) / 3\rceil}\right)$. Nešetril and Poljak [21] showed that the pattern detection problem can be reduced to rectangular matrix multiplication. In particular, when $k \equiv q \bmod 3$, detecting a $k$ node pattern in an $n$ node $G$ can be reduced in $O\left(n^{(2 k+q) / 3}\right)$ time to the product of an $n^{\lfloor k / 3\rfloor} \times n^{\lceil k / 3\rceil}$ matrix by an $n^{\lceil k / 3\rceil} \times n^{\lceil(k-1) / 3\rceil}$ matrix.

Here we first recall the approach from [41], and then generalize the ideas there to obtain an approach for all $k$ to show that (1) for all $k \leq 6$ and for all $k$-node $H$ that is not a Clique or Independent Set, $H$ can be detected in $O(C(n, k-1))$ time, whp, and (2) for all $k \geq 3$, there is a pattern that can be detected in time $O(C(n, k-1))$, whp.

\subsection{The Approach from [41]}

Vassilevska W. et al. [41] proposed the following approach for detecting a copy of $H$ in $G$ :

(1) First obtain a random subgraph $G^{\prime}$ of $G$ by removing each vertex of $G$ independently and uniformly at random with probability $1 / 2$.

(2) Compute a quantity $Q$ that equals the number of induced $H$ in $G^{\prime}$, modulo a particular integer $q$.

(3) If $Q \neq 0 \bmod q$, return that $G$ contains an induced $H$, and otherwise, return that $G$ contains no induced $H$ with high probability.

The following lemma from [41] implies that (for any $q$ ), if $G$ contains a copy of $H$, after the first step, with $O(1)$ probability, the number of copies of $H$ in $G^{\prime}$ is not divisible by $q$.

Lemma 3.1 ([41]). Let $q \geq 2$ be an integer, $G, H$ be undirected graphs. Let $G^{\prime}$ be a random induced subgraph of $G$ such that each vertex is taken with probability $\frac{1}{2}$, independently. If there is at least 
one induced- $H$ in $G$, the number of induced- $H$ in $G^{\prime}$ is not a multiple of $q$ with probability at least $2^{-|H|}$.

Now using Lemma 3.1, we can sample graph $G^{\prime}$ from $G$, and with probability $2^{-k}$ we have the number of induced $H$ is not divisible by $q$. To obtain higher probability, we can simply repeat this procedure.

Hence, it suffices to provide an algorithm for counting the number of copies of $H$ modulo some integer. The approach from [41] is to efficiently compute a quantity which is an integer linear combination $Q=\sum_{i=1}^{t} \alpha_{i} n_{H_{i}}$ of the number of copies $n_{H_{i}}$ in $G$ of several different patterns $H=H_{1}, H_{2}, \ldots, H_{t}$, so that some integer $q$ divides the coefficients $\alpha_{i}$ in front of $n_{H_{i}}$ for $i>1$ but $q$ does not divide $\alpha_{1}$. Thus, $Q=\alpha_{1} n_{H} \bmod q$.

Suppose that $d$ is the largest common divisor of $\alpha_{1}$ and $q$. Suppose that $d \neq 1$. Since $q$ divides every $\alpha_{k}$ with $k>1, d$ must divide all $\alpha_{i}$. Hence, we could just consider $Q / d$ in place of $Q$ before taking things $\bmod q$. Thus wlog $\alpha_{1}$ and $q$ are coprime, and so $\alpha^{-1}$ exists in $\mathbb{Z}_{q}$. Hence, $Q \alpha^{-1}=n_{H} \bmod q$, and we can use this quantity in step 2 of the approach above.

In prior work, the equations $Q$ were obtained carefully for each particular 4 node pattern. In the remaining of this section we provide an outline of a general and principled approach of obtaining such quantities that can be computed in $O(C(n, k-1))$ time for $k \leq 6$.

\subsection{Setup}

As mentioned earlier, two graphs $H$ and $H^{\prime}$ are isomorphic if there is an injective mapping from the vertex set of $H$ onto the vertex set of $H^{\prime}$ so that edges and non-edges are preserved. We will represent this mapping by presenting permutations of the vertices of $H$ and $H^{\prime}$, i.e. for two graphs $H$ and $H^{\prime}$ with vertex orders $H=\left(v_{1}, \ldots, v_{t}\right)$ and $H^{\prime}=\left(w_{1}, \ldots, w_{t}\right)$, we say $H$ maps to $H^{\prime}$ if for each $i$ and $j$, $\left(v_{i}, v_{j}\right) \in E(H)$ if and only if $\left(w_{i}, w_{j}\right) \in E\left(H^{\prime}\right)$. Note that if $H$ maps to $H^{\prime}, H^{\prime}$ maps to $H$ as well. We refer to $k$-node graphs as patterns, and we want to detect them in $n$-node graphs. We will assume that every graph we consider is given with a vertex ordering, unless otherwise specified. We call a pattern with an ordering labeled, and otherwise, the pattern is unlabeled. By the subgraph $\left(v_{1}, \ldots, v_{h}\right)$ in a graph $G$, we mean the subgraph induced by these vertices, with this specified order when considering isomorphisms.

We partition all $k$-node patterns with specified vertex orders (there are $2^{\left(\begin{array}{c}k \\ 2\end{array}\right)}$ many of these) into classes and for each class we count the number of subgraphs in a given graph $G$ which map to one of the graphs in this class. Let $k^{\prime}=\left\lfloor\frac{k-1}{3}\right\rfloor$. For a $k$-node pattern $H=\left(v_{0}, \ldots, v_{k-1}\right)$, define the class of $k$-node patterns $C(H)$ as follows:

Let $F$ be the set of the following pairs of vertices: $\left(v_{0}, v_{1}\right), \ldots\left(v_{0}, v_{k^{\prime}}\right)$ (We sometimes refer to these pairs as the first $k^{\prime}$ edges of $\left.H\right)$. Then $H^{\prime}=\left(w_{0}, \ldots, w_{k-1}\right) \in C(H)$ if for all pairs of vertices $\left(v_{i}, v_{j}\right) \notin F$, we have $\left(v_{i}, v_{j}\right) \in E(H)$ if and only if $\left(w_{i}, w_{j}\right) \in E\left(H^{\prime}\right)$. In other words, all graphs in a class agree on the edge relation except possibly for the pairs in $F$.

Note that for any $H^{\prime} \in C(H)$, we have $C\left(H^{\prime}\right)=C(H)$. So each $k$ node pattern is in exactly one class, which is obtained by changing its first $k^{\prime}$ edges.

\subsection{Outline of the General Approach}

Our goal is to detect an unlabeled pattern by counting the number of patterns in different classes of graphs, which can be done as fast as the fastest algorithm for detecting $k-1$-clique (i.e. $C(n, k-1)$ ). Theorem 3.1 states this result formally and is proved in [13].

Theorem 3.1. Let $G$ be an n-node graph and let $c$ be one of the classes of $k$-node patterns. We can count the number of subgraphs in $G$ which map to a pattern in $c$ in $O(C(n, k-1))=$ $O\left(M\left(n^{\left\lfloor\frac{k-1}{3}\right\rfloor}, n^{\left\lceil\frac{k-1}{3}\right\rceil}, n^{\left\lceil\frac{k-2}{3}\right\rceil}\right)\right)$ time, which is the runtime of the fastest algorithm for detecting $K_{k-1}$.

To see what Theorem 3.1 counts in terms of unlabeled patterns, for a class $c$ define $U(c)$ to be the set of unlabeled patterns for which there is an ordering of vertices that is in class $c$. In [13] we show that for a class $c$ and an unlabaled pattern $\tilde{H} \in U(c)$, there is an integer $b_{\tilde{H}}^{c}$ such that Theorem 3.1 computes the following value:

$$
\sum_{\tilde{H} \in U(c)} b_{\tilde{H}}^{c}|A u t(\tilde{H})| n_{\tilde{H}}
$$

Now we will outline the idea of how to detect unlabeled patterns with graph classes. First let $B_{r}$ be the set of unlabeled patterns $\tilde{H}$ such that $r|| A u t(\tilde{H}) \mid$. Note that we have $K_{k}, \bar{K}_{k} \in B_{r}$ for all $r$ such that $r \mid k$ ! (where $K_{k}$ is the $k$-clique and $\overline{K_{k}}$ is the $k$-Independent set). For a fixed unlabeled pattern $\tilde{H}$ which is not the $k$-Independent Set or the $k$-Clique, the idea is to compute the sums of the form (1) for different pattern classes $c$, such that a linear combination of these sums gives us a sum consisting of only the terms from $\tilde{H}$ and patterns $\tilde{H}^{\prime} \in B_{r}$ for some $r$ such that $r \wedge|A u t(\tilde{H})|$. More specifically, we want to compute a sum of the following form:

$$
|A u t(\tilde{H})| n_{\tilde{H}}+\sum_{\tilde{H}^{\prime} \in B_{r}} d_{\tilde{H}^{\prime}}\left|\operatorname{Aut}\left(\tilde{H}^{\prime}\right)\right| n_{\tilde{H}^{\prime}}
$$

where $d_{\tilde{H}^{\prime}}$ are some integers. Then using the fact that this sum is equal to $|\operatorname{Aut}(\tilde{H})| n_{\tilde{H}}$ modulo $r$, by the approach of Vassilevska W. et al. [41] we can assume with constant probability that $r \Lambda n_{\tilde{H}}$, and hence we can detect $\tilde{H}$ in $G$.

For $k \leq 6$, we show in [13] that we can obtain an equation in the form of 2 for any pattern $\tilde{H}$ that is not a clique or an independent set.

Moreover, using this approach, we can show that for any $k$, there is a pattern that contains a $k-1$-clique and can be detected in $O(C(n, k-1))$ time in an $n$-node graph $G$, which means that the runtime obtained for it is tight, if we assume that the best runtime for detecting $k-1$-clique is $O(C(n, k-1))$. Let $H_{s}^{k}$ be the $k$-node pattern consisting of a $(k-1)$-clique and a vertex adjacent to $s$ vertices of the $(k-1)$-clique. By using the value of $\left|A u t\left(H_{s}\right)\right|$, we prove the following theorem [13].

THeOREM 3.2. For all $k>2$, there is some $s$ where the $k$-node pattern $H_{s}^{k}$ can be detected in $O(C(n, k-1))$ time with high probability.

\section{DETECTING NON-INDUCED DIRECTED CYCLES}

In this Section we analyze an algorithm proposed by Yuster and Zwick [43], obtaining the fastest algorithms for $k$-Cycle detection in sparse directed graphs, to date. 


\subsection{Yuster and Zwick's Algorithm}

We refer to [43] and [13] for a description of Yuster-Zwick's algorithm. Here we only state the definition of its runtime.

$$
\begin{aligned}
P_{i, i+1}^{d}=1 ; \forall j \neq i+1: \\
P_{i, j}^{d}=\min \left\{\begin{array}{l}
P_{i, j-1}^{d}+d_{j-1} \\
P_{i+1, j}^{d}+d_{i+1} \\
\min _{i<r<j} \max \left\{P_{i, r}^{d}, P_{r, j}^{d}, M\left(1-d_{i}, 1-d_{r}, 1-d_{j}\right)\right.
\end{array}\right.
\end{aligned}
$$

For $d=\left(d_{0}, \ldots, d_{k-1}\right)$, define

$$
C_{k}\left(d_{0}, \ldots, d_{k-1}\right)=\min _{0 \leq i<j \leq k-1} \max \left\{P_{i, j}^{d}, P_{j, i}^{d}\right\} .
$$

Yuster-Zwick's algorithm runs in $\tilde{\Theta}\left(m^{c_{k}}\right)$ time, where

$$
c_{k}=\max _{d=\left(d_{0}, \ldots, d_{k-1}\right)} \min \left\{\min _{0 \leq i \leq k-1}\left(2-d_{i}\right), C_{k}\left(d_{0}, \ldots, d_{k-1}\right)\right\} .
$$

Yuster and Zwick were only able to analyze $c_{k}$ for $k \leq 5$. In particular, they showed that $c_{3}=2 \omega /(\omega+1), c_{4}=(4 \omega-1)(2 \omega+$ $1), c_{5}=3 \omega /(\omega+2)$. While they were not able to analyze $c_{k}$ for $k>5$, using extensive numerical experiments, they came up with conjectures about the structure of $c_{k}$ for all odd $k$ and for $k=6$. They did not propose a conjecture for larger even $k$.

Conjecture 2. ${ }^{3}$ For all odd $k \geq 3, c_{k} \leq(k+1) \omega /(2 \omega+k-1)$; if $\omega \leq \frac{2 k}{k-1}, c_{k}=(k+1) \omega /(2 \omega+k-1)$.

Conjecture $3 .^{4}$

$$
c_{6}= \begin{cases}\frac{10 \omega-3}{4 \omega+3}, & \text { if } 2 \leq \omega \leq \frac{13}{6} \\ \frac{22-4 \omega}{17-4 \omega}, & \text { if } \frac{13}{6} \leq \omega \leq \frac{9}{4} \\ \frac{11 \omega-2}{4 \omega+5}, & \text { if } \frac{9}{4} \leq \omega \leq \frac{16}{7} \\ \frac{10-\omega}{7-\omega}, & \text { if } \frac{16}{7} \leq \omega \leq \frac{5}{2}\end{cases}
$$

We prove these conjectures, and in addition prove upper bounds on $c_{k}$ that are tight when $\omega=2$.

\subsection{The Runtime of Yuster-Zwick's Algorithm for Finding $k$-Cycles}

Here we prove Conjectures 3 and 2, and in addition we give bounds for all even $k$ that are tight when $\omega=2$. This proves Theorem 1.3 from the introduction. Due to space limit, we omit certain proofs. The fully detailed proofs are included in [13].

To highlight the result for even cycles for which there wasn't even a conjectured runtime, we split it into its separate theorem:

THEOREM 4.1. For all even $k \geq 4, c_{k} \leq \frac{k \omega-\frac{4}{k}}{2 \omega+k-2-\frac{4}{k}}$. This bound is tight for $\omega=2$.

\footnotetext{
${ }^{3}$ The conjecture given in [43] states that $c_{k}=(k+1) \omega /(2 \omega+k-1)$. However, we discover that $c_{k} \leq 2-\frac{2}{k+1}<\frac{(k+1) \omega}{2 \omega+k-1}$ when $\omega>\frac{2 k}{k-1}$.

${ }^{4}$ The conjecture given in [43] had a slight typo in the first case - the denominator stated there was $(4 \omega+4)$ instead of $(4 \omega+3)$. However, looking at the numerical experiments given to us by Uri Zwick we saw that it should be corrected, and indeed we prove that the corrected version is correct.
}

\subsection{Setup: Basic Lemmas}

Fix arbitrary $0 \leq d_{0}, \cdots, d_{k-1} \leq 1$. For simplicity's sake, we write $P_{i, j}$ and $C_{k}$ for $P_{i, j}^{\left(d_{0}, . ., d_{k-1}\right)}$ and $C_{k}\left(d_{0}, \cdots, d_{k-1}\right)$ when $\left(d_{0}, . ., d_{k-1}\right)$ is fixed. We need to prove $C_{k} \leq B$ for some upper bound $B$ that is a function of $k$ and $\omega$.

Here and in what follows, all indices are considered modulo $k$. Visualizing these indices as $k$ points arranging counterclockwise on a circle would make the following definitions and inequalities more intuitive.

Definition 4.1. For any index $r$, and $\delta \geq 0, r$ is $\delta$-low if $d_{r}<\delta$, and $\delta$-high otherwise.

Definition 4.2. For any two indices $i, j$, let $\ell(i, j)=(j-i+1)$ $(\bmod k)$ and $f(i, j)=\sum_{r=i}^{i+\ell(i, j)-1} d_{r}$. Note that $\ell(i, j) \geq 0$. When $\ell(i, j)=0$ (i.e. $i=j+1), f(i, j)=\sum_{r=j+1}^{j} d_{r}=0$.

Repeatedly applying inequality $P_{w, y}^{d} \leq P_{w, y-1}^{d}+d_{y-1}$, which is derived from Equation (3), gives $P_{w, y}^{d} \leq 1+f(w+1, y-1)$

Lemma 4.1. Suppose that $d_{r} \leq \delta \leq d_{i}, d_{j}$. Let $j_{0} \in\{i, j\}$ such that $d_{j_{0}}=\min \left\{d_{i}, d_{j}\right\}$.

(1) If $P_{j, i}^{d}, P_{i, r}^{d}, P_{r, j}^{d} \leq B$ and $C_{k}\left(d_{0}, \cdots, d_{k-1}\right)>B$ then $M(1-$ $\left.d_{i}, 1-d_{j}, 1-d_{r}\right)>B$

(2) If $M\left(1-d_{i}, 1-d_{j}, 1-d_{r}\right) \geq B \geq \omega(1-\delta)$ then $d_{r}+d_{j_{0}} \leq$ $\omega-B-(\omega-2) \delta \leq 2 \delta$, and $d_{r} \leq \omega-B-(\omega-2) \delta-\delta$.

Lemma 4.1 follows fairly straightforwardly from Eq. (3).

LEMma 4.2. For any two indices $i, j$, and integer $t \leq k-2$. If $d_{i}, d_{j} \geq \delta$ and there are no $t+1$ consecutive $\delta$-low indices $r$ such that $i<r<j$ then $P_{i, j} \leq \max \{1+t \delta, \omega(1-\delta)\}$.

As a consequence, if there are no $t+1$ consecutive $\delta$-low indices then $C_{k} \leq \max \{1+t \delta, \omega(1-\delta)\}$.

Proof. Let $i=i_{0}, i_{1}, \ldots, i_{z}=j$ be the indices within $\{i, i+$ $1, \ldots, j\}$ (indices $\bmod k$ ) such that $d_{i_{b}} \geq \delta$ for each $b \in\{0, \ldots, z\}$. Since there are no consecutive $t+1 \delta$-low indices, for each $b$, $P_{i_{b}, i_{b+1}} \leq 1+t \delta$, using the rule $P_{w, y} \leq P_{w, y-1}+d_{y-1}$. Now we can use the matrix multiplication rule to get $P_{i, j} \leq \max \{1+t \delta, M(1-$ $\delta, 1-\delta, 1-\delta)\}$.

\subsection{Finding Odd Cycles}

Here we prove Yuster and Zwick's conjecture that the exponent $c_{k}$ of the runtime when $k$ is odd and $\omega \leq \frac{2 k}{k-1}$ is $\omega(k+1) /(2 \omega+k-1)$. When $k$ is odd and $\omega>\frac{2 k}{k-1}$, matrix multiplication doesn't seem to help, since [3] gives a faster runtime than $m^{\omega(k+1) /(2 \omega+k-1)}$.

Let $t:=\left\lfloor\frac{k-1}{2}\right\rfloor, h:=k-t-1, \delta \geq 0$ be a parameter to be chosen, $B:=1+t \delta$. Note that $t \leq h \leq t+1$ and $2 h \leq k$. Assume $B \geq \omega(1-\delta)$. We prove $C_{k} \leq B$.

By Lemma 4.2, if there are no $t+1$ consecutive $\delta$-low indices then $C_{k} \leq \max \{1+t \delta, \omega(1-\delta)\}=B$. Now, consider the case when there are at least $t+1$ consecutive $\delta$-low indices. WLOG, we can assume that there exists $s \in[0, h-1]$ such that indices 0 and $s$ are $\delta$-high and indices $r$ are $\delta$-low for all $s+1 \leq r \leq k-1$. That there are at most $s-1<t$ indices $r \in(0, s)$ and Lemma 4.2 implies $P_{0, s} \leq B$. Our proof for upper bounds on $C_{k}$ will proceed as follow: suppose $C_{k}>B$, we use Lemma 4.3 to derive multiple inequalities 
of form $d_{i}+d_{r} \leq \omega-B-(\omega-2) \delta \leq 2 \delta$ where $r \in\{i+t, i-t\}$, then sum these to get $f(R+1, R-1) \leq 2 t \delta$, which implies $C_{k} \leq B$ by Lemma 4.4. Lemma 4.3 below follows from Lemmas 4.2 and 4.1.

Lemma 4.3. Consider indices $i, j, r$ where $0 \leq i \leq j \leq s<r \leq$ $k-1$ and $f(r+1, i-1), f(j+1, r-1) \leq t \delta$. If $C_{k}>B$ then $d_{r} \leq \omega-B-(\omega-2) \delta-\delta$, and $d_{r}+\min \left\{d_{i}, d_{j}\right\} \leq \omega-B-(\omega-2) \delta \leq 2 \delta$.

LEMMA 4.4. If there exists index $R, 0 \leq R \leq k-1$ such that $f(R+1, R-1) \leq 2 t \delta$ then $C_{k} \leq B$

Proof. For every index $r \in[R+1, R-2]: P_{R, r+1}+P_{r, R} \leq$ $(1+f(R+1, r))+(1+f(r+1, R-1))=2+f(R+1, R-1) \leq 2+2 t \delta=2 B$, so either $P_{R, r+1} \leq B$ or $P_{r, R} \leq B$.

Note that $P_{R, R+1}=1 \leq B$, so there exists index $r^{*}:=\max \{r \mid R+$ $\left.1 \leq r \leq R-1 \wedge P_{R, r} \leq B\right\}$. If $r^{*}=R-1$, then $P_{R, R-1} \leq B$ and $P_{R-1, R}=1 \leq B$ so $C_{k} \leq \max \left\{P_{R, R-1}, P_{R-1, R}\right\} \leq B$. If $r^{*} \leq R-2$, then either $P_{R, r+1} \leq B$ or $P_{r, R} \leq B$. By definition of $r^{*}, P_{R, r+1}>B$, so $P_{r, R} \leq B$ and $C_{k} \leq \max \left\{P_{R, r}, P_{r, R}\right\} \leq B$.

To use 4.3, we need Definition 4.3 to ensure the preconditions, and Definition 4.4 to get rid of the $\min \{.,$.$\} symbol.$

Definition 4.3. For any integer $q$, arc $(i, j)$ is $q$-low if $f(i, j) \leq$ $\delta(\ell(i, j)-q)$ and $q$-high otherwise.

We have the following lemmas, which follow straightforwardly from the fact that $d_{i} \leq \delta$ for $i \in[s+1, k-1]$.

Lemma 4.5. Consider indices $i, j$ such that $s+1 \leq i \leq j+1 \leq k$. If $(i, j)$ is $q$-low then $\left(i^{\prime}, j^{\prime}\right)$ is $q$-low for any $s+1 \leq i^{\prime} \leq i$ and $j \leq j^{\prime} \leq k-1$. If $(i, j)$ is $q$-high then $\left(i^{\prime}, j^{\prime}\right)$ is $q$-high for any $i \leq i^{\prime} \leq$ $j^{\prime}+1 \leq j+1$.

Lemma 4.6. For any indices $i, j$ such that $s+1 \leq i \leq j+1 \leq k$, $(i, j)$ is 0 -low. If $C_{k}>B$ then $(s+1, k-1)$ is $h-s$ high, thus so is $(i, j)$.

Definition 4.4. Define sequences $\left(a_{n}\right),\left(b_{n}\right)$ for $n \in\{0, \cdots, s\}$ as follows:

$a_{0}=0, b_{0}=s,\left(a_{n}, b_{n}\right)=\left\{\begin{array}{l}\left(a_{n-1}+1, b_{n-1}\right) \text { if } d_{a_{n}} \leq d_{b_{n}} \\ \left(a_{n-1}, b_{n-1}-1\right) \text { else }\end{array}\right.$

Clearly, $\left(a_{n}\right)$ is weakly increasing, $\left(b_{n}\right)$ is weakly decreasing and $b_{n}-a_{n}=s-n \geq 0$. Let $T:=a_{s}=b_{s}$.

Theorem 4.2. Let $p, q$ be integers in $[0, h-s-1]$. Let $\Delta:=p-q$. For every index $i$, let $i^{\Delta}:=i+\Delta$. Let $m=s-h+t \leq s$. We say condition $(p, q)$ holds if $\left(s+1, b_{m}^{\Delta}+t-1\right)$ is p-low, $\left(s+1, b_{m}^{\Delta}+t\right)$ is $(p+1)$-high, $\left(a_{m}^{\Delta}-t+1, k-1\right)$ is $q$-low and $\left(a_{m}^{\Delta}-t, k-1\right)$ is $(q+1)$-high, and property $(p, q, B)$ holds if condition $(p, q)$ implies $C_{k} \leq B$.

(1) If condition $(p, q)$ holds and $C_{k}>B$ then:

(a) $\forall n \in\{0, . ., m\}$ :

$$
\begin{gathered}
f\left(a_{n}^{\Delta}-t+1, a_{n}-1\right) \leq t \delta \\
f\left(b_{n}+1, b_{n}^{\Delta}+t-1\right) \leq t \delta \\
d_{r}+d_{r^{\Delta}-t} \leq \omega-B-(\omega-2) \delta \leq 2 \delta \forall r, 0 \leq r<a_{n} \\
d_{r}+d_{r^{\Delta}+t} \leq \omega-B-(\omega-2) \delta \leq 2 \delta \forall r, b_{n}<r \leq s
\end{gathered}
$$

(b) $f\left(b_{m}+1, a_{m}-1\right) \leq 2 t \delta$

(2) Property $(p, q, B)$ holds when $k$ is odd i.e. if $k$ is odd and condition $(p, q)$ holds, then $C_{k} \leq B$.

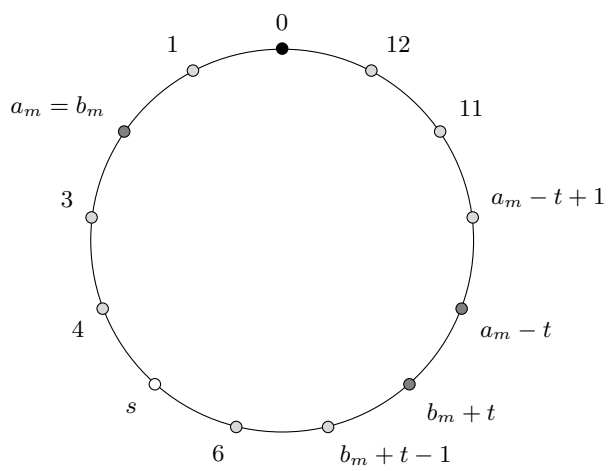

Figure 4: A visualization of the setup of Theorem 4.2 when $k=13, t=h=6, s=h-1=5, \Delta=0$ and $a_{m}=b_{m}=2$.

Proof. We prove 4.2.1(a) by induction on $n$ for $n \in\{0, . ., m\}$. First, observe a useful fact (proof omitted):

FACT 4.1. $\forall n \in\{0, . ., m\}:\left(s+1, b_{n}^{\Delta}+t-1\right)$ is $p$-low, $\left(s+1, a_{n}^{\Delta}-t-1\right)$ is $(p+1)$-high, $\left(a_{n}^{\Delta}-t+1, k-1\right)$ is $q$-low, $\left(b_{n}^{\Delta}+t+1, k-1\right)$ is $(q+1)$-high.

Now, let us proceed with the inductive proof.

Base case, $n=0$ :

By Fact $4.1,\left(s+1, b_{0}^{\Delta}+t-1\right)$ is $p$-low, thus also $\Delta$-low since $p \geq \Delta$, so:

$$
f\left(s+1, b_{0}^{\Delta}+t-1\right) \leq \delta\left(\ell\left(s+1, b_{0}^{\Delta}+t-1\right)-\Delta\right)<t \delta
$$

Symmetrically, $\left(a_{0}^{\Delta}-t+1, k-1\right)$ is $-\Delta$-low, so:

$$
f\left(a_{0}^{\Delta}-t+1, k-1\right) \leq \delta\left(\ell\left(a_{0}^{\Delta}-t+1, a_{0}-1\right)-(-\Delta)\right)<t \delta
$$

Since $a_{0}=0$ and $b_{0}=s$, inequalities (5) and (6) are proved. Inequalities (7) and (8) are trivially true.

Suppose 4.2.1(a) is true for $n-1$ where $m \geq n \geq 1$. WLOG, assume $d_{a_{n-1}} \leq d_{b_{n-1}}$. The case $d_{a_{n-1}}>d_{b_{n-1}}$ is analogous.

By Definition 4.4, $a_{n}=a_{n-1}+1, b_{n}=b_{n-1}$. Thanks to inductive assumption, we only need to show:

$$
\begin{gathered}
d_{a_{n-1}}+d_{a_{n-1}^{\Delta}-t} \leq \omega-B-(\omega-2) \delta, \text { and } \\
f\left(a_{n}^{\Delta}-t+1, a_{n}-1\right) \leq t \delta .
\end{gathered}
$$

Below, write $R$ in place of $a_{n-1}^{\Delta}-t=a_{n}^{\Delta}-t-1=a_{n}^{\Delta}+h$ for simplicity's sake.

By the inductive assumption, $f\left(R+1, a_{n-1}-1\right)=f\left(a_{n-1}^{\Delta}-t+\right.$ $\left.1, a_{n-1}-1\right) \leq t \delta$ and $f\left(b_{n-1}+1, b_{n-1}^{\Delta}+t-1\right) \leq t \delta$. Observe that $R-1 \in\left[b_{n-1}, b_{n-1}^{\Delta}+t-1\right]$, so $f\left(b_{n-1}+1, R-1\right) \leq f\left(b_{n-1}+1, b_{n-1}^{\Delta}+\right.$ $t-1) \leq t \delta$. By Lemma 4.3, $d_{a_{n-1}}+d_{R} \leq \omega-B-(\omega-2) \delta \leq 2 \delta$.

By the inductive assumption and above statement, $\forall 0 \leq i \leq$ $a_{n-1}: d_{i} \leq 2 \delta-d_{i^{\Delta}-t}$. Hence:

$$
f\left(0, a_{n-1}\right) \leq \sum_{i=0}^{a_{n-1}}\left(2 \delta-d_{i^{\Delta}-t}\right)=2 \ell\left(0, a_{n-1}\right) \delta-f\left(0^{\Delta}-t, R\right)
$$

Recall that $a_{n}=a_{n-1}+1$, so $R=a_{n-1}^{\Delta}-t=a_{n}^{\Delta}-t-1$. By Fact 4.1, $(s+1, R)$ is $(p+1)$-high. Since $0^{\Delta}-t \geq k+(s+1-h)-t \geq s+1$, by Lemma $4.5,\left(0^{\Delta}-t, R\right)$ is also $(p+1)$-high. Thus, $f\left(0, a_{n-1}\right) \leq$ $\delta\left[2 \ell\left(0, a_{n-1}\right)-\left(\ell\left(0^{\Delta}-t, a_{n-1}^{\Delta}-t\right)-(p+1)\right)\right]$. Again by Fact 4.1, 
$\left(a_{n}^{\Delta}-t+1, k-1\right)$ is $q$-low. This combined with above equation implies:

$$
f\left(a_{n}^{\Delta}-t+1, a_{n}-1\right)=f\left(a_{n}^{\Delta}-t+1, k-1\right)+f\left(0, a_{n-1}\right) \leq t \delta .
$$

where the simplification follows from $a_{n}=a_{n-1}+1$ and $\Delta=p-q$. Hence, 4.2.1(a) is still true for $n$, so true for all $n \in\{0, \ldots, m\}$.

Now, we prove 4.2.1(b). Note that $b_{m}^{\Delta}-a_{m}^{\Delta}=b_{m}-a_{m}=s-m=$ $h-t$, so $b_{m}^{\Delta}+t=a_{m}^{\Delta}+h$. By 4.2.1(a)'s (7), (8):

$$
\begin{aligned}
2 \delta m & \geq \sum_{i=0}^{a_{m}-1}\left(d_{i}+d_{i^{\Delta}-t}\right)+\sum_{j=b_{m}+1}^{s}\left(d_{j}+d_{j^{\Delta}+t}\right) \\
& =f\left(0, a_{m}-1\right)+f\left(b_{m}+1, s\right)+f\left(h^{\Delta}+1, s^{\Delta}+t\right)
\end{aligned}
$$

That $|\Delta| \leq h-s-1,2 h \leq k$ and $h-1 \leq t$ implies $h^{\Delta} \in[s+1, k-1]$ and $s^{\Delta}+t+1 \in[s+1, k-1]$. By Lemma 4.6:

$$
\begin{aligned}
f\left(s+1, h^{\Delta}\right) & \leq \ell\left(s+1, h^{\Delta}\right) \delta=\left(h^{\Delta}-s\right) \delta \\
f\left(s^{\Delta}+t+1, k-1\right) & \leq \ell\left(s^{\Delta}+t+1, k-1\right) \delta=\left(h-s^{\Delta}\right) \delta
\end{aligned}
$$

Sum equations in (11) with equation (10), and rearrange terms, we get $2 t \delta \geq f\left(b_{m}+1, a_{m}-1\right)$

Now, we prove 4.2.2. When $k$ is odd, $h=t, m=s$, and $a_{m}=$ $b_{m}=a_{s}=b_{s}=T$. Suppose that $C_{k}>B$, then $f(T+1, T-1) \leq 2 t \delta$ by $4.2 .1(\mathrm{~b})$, thus $C_{k} \leq B$ by lemma 4.4 .

To conclude, we prove that property $(p, q, B)$ implies $C_{k} \leq B$.

Lemma 4.7. Suppose that property $(p, q, B)$ holds $\forall 0 \leq p, q \leq$ $h-s-1$. Then:

(1) Let $p, q, \Delta$ be integers such that $0 \leq p, q \leq h-s-1$, and $\Delta=p-q$. If $\left(s+1, b_{m}^{\Delta}+t-1\right)$ is $p$-low and $\left(a_{m}^{\Delta}-t, k-1\right)$ is $(q+1)$ high, then $C_{k} \leq B$. Analogously, if $\left(a_{m}^{\Delta}-t+1, k-1\right)$ is $q$-low and $\left(s+1, b_{m}^{\Delta}+t\right)$ is $(p+1)$-high then $C_{k} \leq B$.

(2) $C_{k} \leq B$.

Proof. (1) We prove the first statement by induction on $\Delta=p-q$ where $|\Delta| \leq h-s-1$. The second one follows by symmetry.

Base case: $\Delta=h-s-1$. Since $0 \leq p, q \leq h-s-1, p=h-s-1, q=0$. By Lemma $4.6,\left(s+1, b_{m}^{\Delta}+t\right)$ is $(p+1)$-high and $\left(a_{m}^{\Delta}-t+1, k-1\right)$ is $q$-low, so $C_{k} \leq B$ because property $(p, q, B)$ holds.

Suppose Lemma 4.7 is true for $\Delta^{\prime}=\Delta+1$, we prove it is also true for $\Delta$. $\forall$ index $i$, let $i^{\Delta^{\prime}}:=i+\Delta^{\prime}=i^{\Delta}+1$.

If $\left(s+1, b_{m}^{\Delta}+t\right)=\left(s+1, b_{m}^{\Delta^{\prime}}+t-1\right)$ is $(p+1)$-low: Since $a_{m}^{\Delta^{\prime}}-t>a_{m}^{\Delta}-t$ and $\left(a_{m}^{\Delta}-t, k-1\right)$ is $(q+1)$-high, by Lemma 4.5, $\left(a_{m}^{\Delta^{\prime}}-t, k-1\right)$ is $(q+1)$-high. Apply induction's assumption for $\Delta^{\prime}=p+1-q$, we have $C_{k} \leq B$.

When $\left(a_{m}^{\Delta}-t+1, k-1\right)=\left(a_{m}^{\Delta^{\prime}}-t, k-1\right)$ is $q$-high, we use an analogous argument, and the inductive assumption for $\Delta^{\prime}=$ $p-(q-1)$ to obtain $C_{k} \leq B$.

The remaining case is $\left(s+1, b_{m}^{\Delta}+t\right)$ is $(p+1)$-high and $\left(a_{m}^{\Delta}-t+\right.$ $1, k-1)$ is $q$-low, thus condition $(p, q)$ holds. Then $C_{k} \leq B$ because of property $(p, q, B)$.

(2) We now prove property (2). Suppose for contradiction that $C_{k}>B$. By Lemma 4.6, there exists $q \in \mathbb{N} \cap[0, h-s-1]$ such that $\left(a_{m}-t, k-1\right)$ is $(q+1)$-high and $q$-low.

If $\left(s+1, b_{m}+t\right)$ is $(q+1)$-low: Since $\left(a_{m}-t, k-1\right)$ is $(q+1)$-high, so is $\left(a_{m}-t+1, k-1\right)$ by Lemma 4.5 . Let $p=q+1, \Delta=p-q=1$. Since $\left(s+1, b_{m}^{\Delta}+t-1\right)=\left(s+1, b_{m}+t\right)$ is $p$-low, $\left(a_{m}^{\Delta}-t, k-1\right)=$
Table 1: Hard-case degree class, for $B=c_{6}(\omega)$ and $\delta=\frac{B-1}{2}$

\begin{tabular}{|c|c|c|c|c|c|c|}
\hline$\omega$ & $d_{0}$ & $d_{1}$ & $d_{2}$ & $d_{3}$ & $d_{4}$ & $d_{5}$ \\
\hline$\in\left[2, \frac{13}{6}\right]$ & $\frac{4 \delta}{3}$ & $\delta$ & $\delta$ & $\frac{2 \delta}{3}$ & $\frac{2 \delta}{3}$ & $\frac{2 \delta}{3}$ \\
\hline$\in\left[\frac{13}{6}, \frac{9}{4}\right]$ & $2-B$ & $\frac{7 B-10}{4}$ & $\frac{6-3 B}{4}$ & $\frac{2-B}{2}$ & $\frac{2-B}{2}$ & $2 B-3$ \\
\hline$\in\left[\frac{9}{4}, \frac{16}{7}\right]$ & $\frac{8 \delta}{7}$ & $\frac{8 \delta}{7}$ & $\frac{6 \delta}{7}$ & $\frac{4 \delta}{7}$ & $\frac{4 \delta}{7}$ & $\frac{6 \delta}{7}$ \\
\hline$\in\left[\frac{16}{7}, \frac{5}{2}\right]$ & $2-B$ & $2-B$ & $2 B-3$ & $\frac{2-B}{2}$ & $\frac{2-B}{2}$ & $2 B-3$ \\
\hline
\end{tabular}

$\left(a_{m}-t+1, k-1\right)$ is $(q+1)$-high, $C_{k} \leq B$ by Sublemma 4.7.(1) (contradiction).

So $\left(s+1, b_{m}+t\right)$ is $(q+1)$-high. We can similarly prove $\left(s+1, b_{m}+\right.$ $t-1)$ is $q$-low and $\left(a_{m}-t+1, k-1\right)$ is $q$-low. These 3 conditions, along with $\left(a_{m}-t, k-1\right)$ is $(q+1)$-high, implies condition $(q, q)$, and thus $C_{k} \leq B$ by property $(q, q, B)$.

Now, we return to the assumption that $k$ is odd. Set $B:=1+t \delta=$ $\omega(1-\delta)=(k+1) \omega /(2 \omega+k-1)$. Lemma 4.7 and 4.2.2 together imply $C_{k} \leq B$. Since the choice $d_{0}, \cdots, d_{k-1}$ is arbitrary, $c_{k} \leq B$. We show the bound is tight and that $c_{k}=B$ for some choice of the degrees. Assume $\omega \leq \frac{2 k}{k-1}$. Set $d_{0}=\cdots=d_{k-1}=\delta$. Easy to check, via Eq. (3) that $C_{k}(\delta, \cdots, \delta)=\omega(k+1) /(2 \omega+k-1)$.We term $(\delta, \cdots, \delta)$ the "hard-case degree class" i.e. the degree tuple that produces a runtime matching our upper bound.

\subsection{Finding 6-Cycles}

Here we analyze discuss Conjecture 3 on the runtime of YusterZwick's algorithm for finding 6-Cycles. Its (omitted) proof, which is basically a case analysis, follows the same technique in Section 4.4 i.e. assuming $C_{k}$ is greater than a certain bound, we can derive certain inequalities Equation (3), that together leads to a contradiction. The trick is to figure out "hard-case degree class(es)" (summarized in Table 1), then combining equations from (3) that is compatible with these degree values.

\subsection{Finding Even Cycles}

When $k$ is even, $h=t+1$. Let $\beta:=\delta \frac{t}{h}<\delta$, then $B=1+t \delta=1+h \beta$. Set $B=(\omega-2)(1-\delta)+(1-\delta)+(1-\beta) \geq \omega(1-\delta)$. Note that, $B=\frac{k \omega-\frac{4}{k}}{2 \omega+k-2-\frac{4}{k}}$. We will prove $C_{k}:=C_{k}\left(d_{0}, \cdots, d_{k-1}\right) \leq B$. Define $s,\left(a_{n}\right),\left(b_{n}\right)$ same as in Subsection 4.4. We will sum inequalities of the form $d_{i}+d_{r} \leq \delta+\beta$ to get $f\left(b_{s}+1, a_{s}-1\right) \leq t \delta+h \beta=2 t \delta$, then use Lemma 4.4 and 4.7 to conclude that $C_{k} \leq B$. Due to space constrained, we omit the proof. Our analysis is tight for $\omega=2$, since there is a "hard-case degree class": $d_{0}=2 \beta, d_{1}=\cdots=d_{t}=$ $\delta, d_{t+1}=\cdots=d_{k-1}=\beta$.

\section{ACKNOWLEDGMENTS}

We would like to thank Uri Zwick for pointing us to the open problem of analyzing the Yuster-Zwick algorithm.

\section{REFERENCES}

[1] Amir Abboud, Arturs Backurs, and Virginia Vassilevska Williams. If the current clique algorithms are optimal, so is valiant's parser. In IEEE 56th Annual Symposium on Foundations of Computer Science, FOCS 2015, Berkeley, CA, USA, 17-20 October, 2015, pages 98-117, 2015. 
[2] N. Alon, P. Dao, I. Hajirasouliha, F. Hormozdiari, and S. Cenk Sahinalp. Biomolecular network motif counting and discovery by color coding. Bioinformatics, 24(13):i241-i249, 2008.

[3] N. Alon, R. Yuster, and U. Zwick. Finding and counting given length cycles. Algorithmica, 17:209-223, 1997.

[4] Markus Bläser, Balagopal Komarath, and Karteek Sreenivasaiah. Graph pattern polynomials. CoRR, abs/1809.08858, 2018.

[5] B. Bollobás. The chromatic number of random graphs. Combinatorica, 8(1):49-55, 1988.

[6] B. Bollobás, P.A. Catlin, and P. Erdös. Hadwiger's conjecture is true for almost every graph. European fournal of Combinatorics, 1(3):195 - 199, 1980.

[7] A. Bondy and M. Simonovits. Cycles of even length in graphs. Journal of Combinatorial Theory, 16:97-105, 1974.

[8] Karl Bringmann and Philip Wellnitz. Clique-based lower bounds for parsing tree-adjoining grammars. In 28th Annual Symposium on Combinatorial Pattern Matching, CPM 2017, July 4-6, 2017, Warsaw, Poland, pages 12:1-12:14, 2017.

[9] Jianer Chen, Benny Chor, Mike Fellows, Xiuzhen Huang, David W. Juedes, Iyad A Kanj, and Ge Xia. Tight lower bounds for certain parameterized np-hard problems. Inf. Comput., 201(2):216-231, 2005.

[10] D. Corneil, Y. Perl, and L. Stewart. A linear recognition algorithm for cographs. SIAM fournal on Computing, 14(4):926-934, 1985

[11] Radu Curticapean, Holger Dell, and Dániel Marx. Homomorphisms are a good basis for counting small subgraphs. In Proceedings of the 49th Annual ACM SIGACT Symposium on Theory of Computing, STOC 2017, Montreal, QC, Canada, June 19-23, 2017, pages 210-223, 2017.

[12] Søren Dahlgaard, Mathias Bæk Tejs Knudsen, and Morten Stöckel. Finding even cycles faster via capped k-walks. In Proceedings of the 49th Annual ACM SIGACT Symposium on Theory of Computing, STOC 2017, Montreal, QC, Canada, June 19-23, 2017, pages 112-120, 2017.

[13] Mina Dalirrooyfard, Thuy Duong Vuong, and Virginia Vassilevska Williams. Graph pattern detection: Hardness for all induced patterns and faster non-induced cycles. CoRR, abs/1904.03741, 2019.

[14] F. Eisenbrand and F. Grandoni. On the complexity of fixed parameter clique and dominating set. Theor. Comp. Sci., 326(1-3):57-67, 2004.

[15] Peter Floderus, Miroslaw Kowaluk, Andrzej Lingas, and Eva-Marta Lundell. Detecting and counting small pattern graphs. In Algorithms and Computation - 24th International Symposium, ISAAC 2013, Hong Kong, China, December 16-18, 2013, Proceedings, pages 547-557, 2013.

[16] Peter Floderus, Miroslaw Kowaluk, Andrzej Lingas, and Eva-Marta Lundell. In duced subgraph isomorphism: Are some patterns substantially easier than others? Theor. Comput. Sci., 605:119-128, 2015.

[17] Fedor V. Fomin, Daniel Lokshtanov, Venkatesh Raman, Saket Saurabh, and B. V. Raghavendra Rao. Faster algorithms for finding and counting subgraphs. F. Comput. Syst. Sci., 78(3):698-706, 2012.

[18] Francois Le Gall and Florent Urrutia. Improved rectangular matrix multiplication using powers of the coppersmith-winograd tensor. In Proceedings of the TwentyNinth Annual ACM-SIAM Symposium on Discrete Algorithms, SODA 2018, New Orleans, LA, USA, January 7-10, 2018, pages 1029-1046, 2018.

[19] H. Hadwiger. Ungelöste probleme nr. 20. Elemente der Mathematik, 12:121, 1957.

[20] Russell Impagliazzo and Ramamohan Paturi. On the complexity of k-sat. $f$ Comput. Syst. Sci., 62(2):367-375, 2001.

[21] A. Itai and M. Rodeh. Finding a minimum circuit in a graph. SIAM f. Computing, 7(4):413-423, 1978.
[22] Ken-ichi Kawarabayashi and Bjarne Toft. Any 7-chromatic graphs has $K_{7}$ or $K_{4,4}$ as A minor. Combinatorica, 25(3):327-353, 2005.

[23] T. Kloks, D. Kratsch, and H. Müller. Finding and counting small induced subgraphs efficiently. Inf. Proc. Letters, 74(3-4):115-121, 2000.

[24] Miroslaw Kowaluk and Andrzej Lingas. A fast deterministic detection of small pattern graphs in graphs without large cliques. In WALCOM: Algorithms and Computation, 11th International Conference and Workshops, WALCOM 2017, Hsinchu, Taiwan, March 29-31, 2017, Proceedings., pages 217-227, 2017.

[25] Miroslaw Kowaluk, Andrzej Lingas, and Eva-Marta Lundell. Counting and detecting small subgraphs via equations. SIAM J. Discrete Math., 27(2):892-909, 2013.

[26] François Le Gall. Powers of tensors and fast matrix multiplication. In International Symposium on Symbolic and Algebraic Computation, ISSAC '14, Kobe, Japan, July 23-25, 2014, pages 296-303, 2014.

[27] Andrea Lincoln, Virginia Vassilevska Williams, and Ryan Williams. Tight hardness for shortest cycles and paths in sparse graphs. In Proceedings of the TwentyNinth Annual ACM-SIAM Symposium on Discrete Algorithms, SODA '18, pages 1236-1252, 2018.

[28] B. Monien. How to find long paths efficiently. Annals of Discrete Mathematics, 25:239 - 254, 1985.

[29] J. Nešetřil and S. Poljak. On the complexity of the subgraph problem. Commentationes Math. Universitatis Carolinae, 26(2):415-419, 1985.

[30] Stephan Olariu. A simple linear-time algorithm for computing the center of an interval graph. International fournal of Computer Mathematics, 34:121-128, 1990.

[31] N. Przulj, D. G. Corneil, and I. Jurisica. Efficient estimation of graphlet frequency distributions in protein-protein interaction networks. Bioinformatics, 22(8):974980, 2006.

[32] Neil Robertson, Daniel Sanders, Paul Seymour, and Robin Thomas. The fourcolour theorem. fournal of Combinatorial Theory, Series B, 70(1):2 - 44, 1997.

[33] Neil Robertson, Paul Seymour, and Robin Thomas. Hadwiger's conjecture for k6-free graphs. Combinatorica, 13(3):279-361, 1993.

[34] Benjamin Rossman. On the constant-depth complexity of k-clique. In Proceedings of the Fortieth Annual ACM Symposium on Theory of Computing, STOC '08, pages 721-730, 2008.

[35] V. Vassilevska and R. Williams. Finding, minimizing, and counting weighted subgraphs. In Proc. STOC, pages 455-464, 2009.

[36] Virginia Vassilevska. Efficient algorithms for clique problems. Inf. Process. Lett., 109(4):254-257, 2009.

[37] V. Vassilevska Williams and R. Williams. Subcubic equivalences between path, matrix and triangle problems. In Proc. FOCS, pages 645-654, 2010.

[38] Virginia Vassilevska Williams. Efficient algorithms for path problems in weighted graphs. Ph.D. Thesis, Carnegie Mellon University, 2008.

[39] Virginia Vassilevska Williams. Multiplying matrices faster than CoppersmithWinograd. In Proceedings of the 44th Symposium on Theory of Computing Conference, STOC 2012, New York, NY, USA, May 19 - 22, 2012, pages 887-898, 2012.

[40] K. Wagner. Über eine eigenschaft der ebenen komplexe. Mathematische Annalen, 114(1):570-590, 1937

[41] Virginia Vassilevska Williams, Joshua R. Wang, Richard Ryan Williams, and Huacheng Yu. Finding four-node subgraphs in triangle time. In Proceedings of the Twenty-Sixth Annual ACM-SIAM Symposium on Discrete Algorithms, SODA 2015, San Diego, CA, USA, January 4-6, 2015, pages 1671-1680, 2015.

[42] R. Yuster and U. Zwick. Finding even cycles even faster. In Proc. ICALP, pages 532-543, 1994

[43] R. Yuster and U. Zwick. Detecting short directed cycles using rectangular matrix multiplication and dynamic programming. In Proc. SODA, pages 247-253, 2004. 